\title{
FOXE3 mutations predispose to thoracic aortic aneurysms and dissections
}

\author{
Shao-Qing Kuang, ${ }^{1}$ Olga Medina-Martinez, ${ }^{2}$ Dong-chuan Guo, ${ }^{1}$ Limin Gong, ${ }^{1}$ Ellen S. Regalado, ${ }^{1}$ Corey L. Reynolds, ${ }^{3}$ \\ Catherine Boileau, ${ }^{4}$ Guillaume Jondeau, ${ }^{5}$ Siddharth K. Prakash, ${ }^{1}$ Callie S. Kwartler, ${ }^{1}$ Lawrence Yang Zhu, ${ }^{1}$ Andrew M. Peters, ${ }^{1}$ \\ Xue-Yan Duan, ${ }^{1}$ National Registry of Genetically Triggered Thoracic Aortic Aneurysms and Cardiovascular Conditions (GenTAC) \\ Investigators, National Heart, Lung, and Blood Institute (NHLBI) Grand Opportunity (GO) Exome Sequencing Project (ESP), ${ }^{6}$ \\ Michael J. Bamshad, ${ }^{7}$ Jay Shendure, ${ }^{7}$ Debbie A. Nickerson, ${ }^{7}$ Regie L. Santos-Cortez, ${ }^{8}$ Xiurong Dong, ${ }^{9}$ Suzanne M. Leal, ${ }^{8}$ \\ Mark W. Majesky, ${ }^{,}$Eric C. Swindell, ${ }^{10}$ Milan Jamrich, ${ }^{2}$ and Dianna M. Milewicz ${ }^{1}$
}

'Department of Internal Medicine, University of Texas Health Science Center at Houston, Houston, Texas, USA. ²Department of Molecular and Cellular Biology and ${ }^{3}$ Mouse Phenotyping Core, Baylor College of Medicine, Houston, Texas, USA. "AP-HP, Hôpital Bichat, Centre National de Référence pour le syndrome de Marfan et apparentés, Paris, France; Université Paris 7, Paris, France; INSERM, U1148,

Paris, France. ${ }^{5}$ AP-HP, Hôpital Bichat, Centre National de Référence pour le Syndrome de Marfan et apparentés, Paris, France; Université Paris 7, Paris, France; AP-HP, Hôpital Bichat, Service de Cardiologie, Paris, France. ${ }^{6}$ The GenTAC Investigators and NHLBI GO ESP are detailed in the Supplemental Acknowledgments. 'Department of Cenome Sciences, University of Washington, Seattle, Washington, USA. ${ }^{8}$ Center for Statistical Genetics, Department of Molecular and Human Genetics, Baylor College of Medicine, Houston, Texas, USA. ${ }^{9}$ Center for Developmental Biology and Regenerative Medicine, Seattle Children's Research Institute, Departments of Pediatrics and Pathology, University of Washington, Seattle, Washington, USA. ${ }^{10}$ Department of Pediatrics, University of Texas Health Science Center at Houston, Houston, Texas, USA.

\begin{abstract}
The ascending thoracic aorta is designed to withstand biomechanical forces from pulsatile blood. Thoracic aortic aneurysms and acute aortic dissections (TAADs) occur as a result of genetically triggered defects in aortic structure and a dysfunctional response to these forces. Here, we describe mutations in the forkhead transcription factor FOXE3 that predispose mutation-bearing individuals to TAAD. We performed exome sequencing of a large family with multiple members with TAADs and identified a rare variant in FOXE3 with an altered amino acid in the DNA-binding domain (p.Asp153His) that segregated with disease in this family. Additional pathogenic FOXE3 variants were identified in unrelated TAAD families. In mice, Foxe3 deficiency reduced smooth muscle cell (SMC) density and impaired SMC differentiation in the ascending aorta. Foxe3 expression was induced in aortic SMCs after transverse aortic constriction, and Foxe 3 deficiency increased SMC apoptosis and ascending aortic rupture with increased aortic pressure. These phenotypes were rescued by inhibiting $\mathrm{p} 53$ activity, either by administration of a p53 inhibitor (pifithrin- $\alpha$ ), or by crossing Foxe $^{-/-}$mice with $p 53^{-/-}$mice. Our data demonstrate that FOXE3 mutations lead to a reduced number of aortic SMCs during development and increased SMC apoptosis in the ascending aorta in response to increased biomechanical forces, thus defining an additional molecular pathway that leads to familial thoracic aortic disease.
\end{abstract}

\section{Introduction}

The major diseases affecting the ascending thoracic aorta are thoracic aortic aneurysms and acute aortic dissections (TAADs). The natural history of a thoracic aortic aneurysm involves progressive enlargement until an acute tear in the inner layer of the aorta causes blood to dissect through the medial layer. The thick medial layer of the aorta is made up of more than 50 layers of alternating elastic lamellae and smooth muscle cells (SMCs) in humans, and the deposition of elastin occurs mostly during development (1). SMCs in the ascending thoracic aorta that synthesize and organize the elastin fibers arise from neural crest (NC) cells, whereas SMCs in the descending aorta are derived from the presomitic mesoderm (2-4).

Heterozygous mutations in genes that have been shown or are predicted to disrupt SMC contraction, decouple SMCs from the extracellular matrix, or impair the function of proteins involved

Conflict of interest: The authors have declared that no conflict of interest exists. Submitted: July 22, 2015; Accepted: December 10, 2015.

Reference information: / Clin Invest. 2016;126(3):948-961. doi:10.1172/JCI83778. in canonical TGF- $\beta$ signaling predispose individuals to familial TAADs (FTAADs) (5). These mutations create a cascade of anatomic and physiologic defects that lead to degeneration of the medial layer of the aorta, characterized by loss or fragmentation of elastic fibers, focal loss of SMCs, accumulation of proteoglycans, and ultimately TAAD $(5,6)$. Currently, 11 genes identified in FTAADs are responsible for only $25 \%$ of the disease incidence in these families. Thus, the causative gene has not been identified in the majority of families with inherited thoracic aortic disease.

\section{Results}

FOXE3 mutations predispose to TAADs. A large family of European descent, TAA337, was identified with 8 cases of acute aortic dissections inherited in an autosomal-dominant manner (Figure 1A). These dissections occurred at an average age of 45.6 years (range 27-63 years) in individuals who also had no history of hypertension. Affected family members presented with acute ascending aortic dissection (type A) with dilatation noted at the time of surgery (except in 1 family member, III:2, who had prior normal aortic 
A

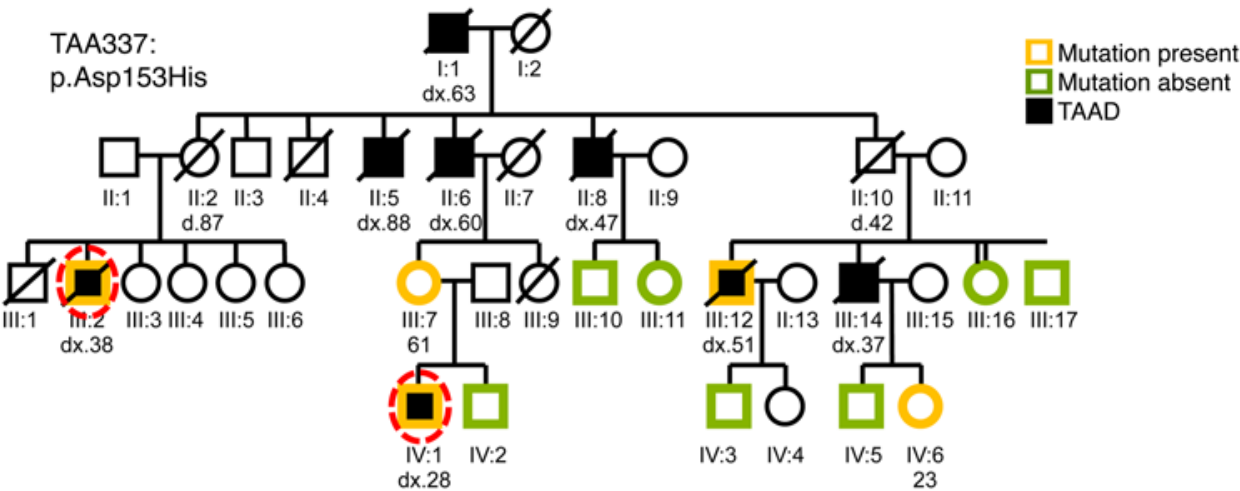

B
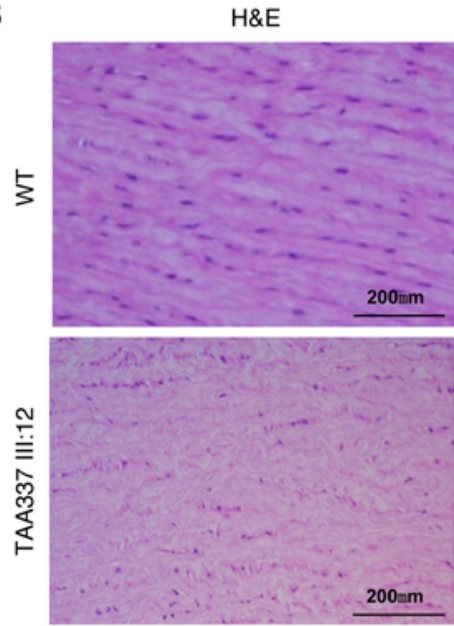

Movat

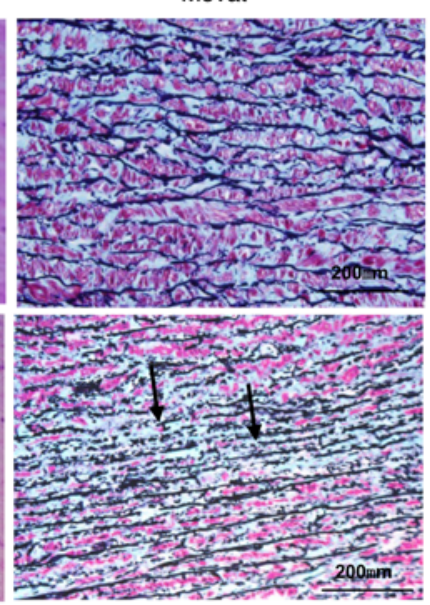

D
FAAD

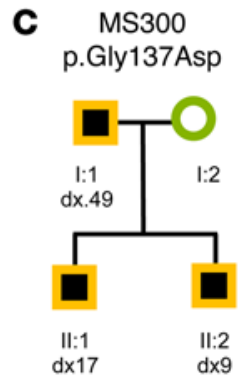

Ser300Gly

।

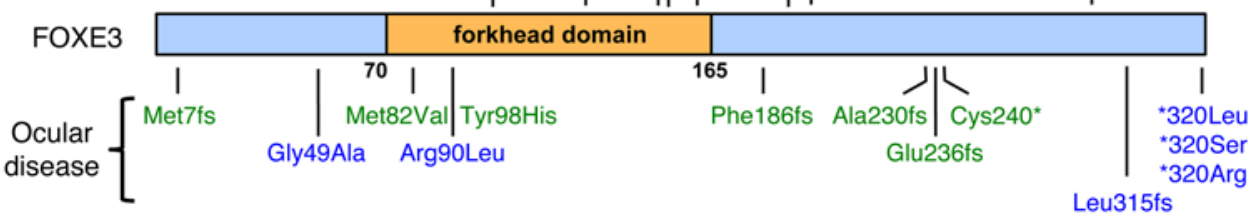

Figure 1. Identification of FOXE3 mutations as a cause of FTAADs.

(A) Pedigree of family TAA337, with the legend showing the designation of the disease and mutation status of the family members. The age at diagnosis of aortic root enlargement and/or dissection (dx.) is shown in years, and "d." indicates age at death. The diagonal line across the symbol indicates that the individual is deceased. DNA from the individuals circled in red was used for exome sequencing. (B) Ascending aortic pathology in individual III:12 from family TAA337 and control aorta. H\&E and Movat pentachrome staining of ascending aortic media from III:12 demonstrated loss of SMCs (red in Movat stain) between intact elastin fibers (black; original magnification, $\times 400$ ). (C) Pedigree of MS300 with a FOXE3 p.Gly137Ala mutation. (D) Schematic representation of FOXE3 protein domain structure and location of rare variants. The FOXE3 rare variants identified in this study are on the top of the protein diagram. Red color indicates variants likely to be pathogenic for FTAAD. The FOXE3 mutations that were reported to be causative for ocular lens abnormalities are on the bottom of protein diagram. Blue color indicates dominant mutations; green color indicates recessive mutations. imaging); only 1 family member presented with a descending aortic dissection (type B; IV:1). Evidence of decreased penetrance in women was supported by the fact that only men were affected; 2 women who inherited the defective gene had no aortic disease (II:2 died in her 80s and III:7 is 61 years of age and asymptomatic). Histologic examination of the aortae from the affected family members showed a unique pathology, in that regions of the medial layer had relatively intact elastin lamellae but loss of SMCs between the lamellae (Figure 1B, arrows, and ref. 7). The known FTAAD genes were excluded as the cause of the inherited aortic disease in this family, and exome sequencing was pursued using DNA samples from 2 distantly related affected individuals (coefficient of relationship =1/16; circles, Figure $1 \mathrm{~A}$ ). The sequencing data were filtered to identify heterozygous variants with a minor allele frequency (MAF) of less than $0.05 \%$ in the Exome Variant Server of the NHLBI GO ESP and shared between affected relatives $(8,9)$. DNA from additional family members was sequenced, and only
1 rare variant, FOXE3 [MIM 601094] c.457G>C (p.Asp153His), segregated with aortic disease in TAA337 (Figure 1A). This variant is present once in 13,000 chromosomes in the ESP database and disrupts an evolutionarily conserved amino acid. Segregation of the FOXE3 variant with aortic disease in this family revealed a lod score of 3.00. Exome and Sanger sequencing of 564 unrelated FTAAD probands identified 7 additional FOXE3 rare variants predicted to disrupt the protein: p.Pro112Ser, p.Gly137Asp, p.Asp156Asn, p.Arg164Ser, p.Gly196Ala, p.Pro202Leu, and p.Ser300Gly (Supplemental Table 1; supplemental material available online with this article; doi:10.1172/JCI83778DS1). Variant p.Gly137Asp was identified in affected members of a small family with aortic root dilatation (Figure 1C); no additional samples were available from the other families. The FOXE3 variants p.Gly137Asp, p.Asp153His, p.Asp156Asn, and p.Arg164Ser are predicted to be damaging or deleterious by at least 4 of 6 functional prediction programs, have a combined annotation-depen- 
A
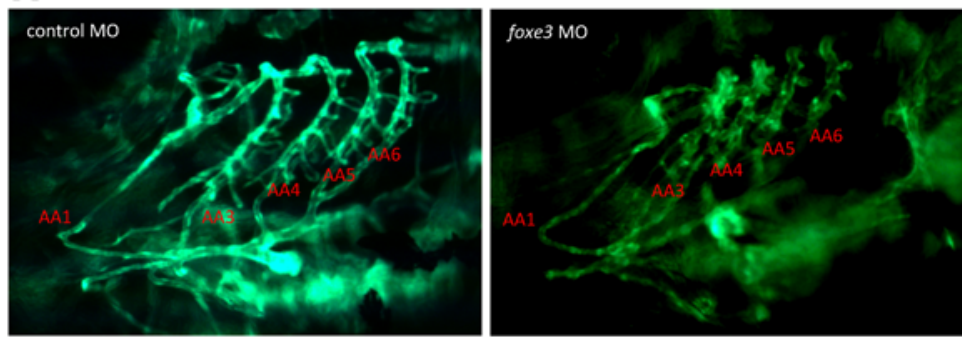

B

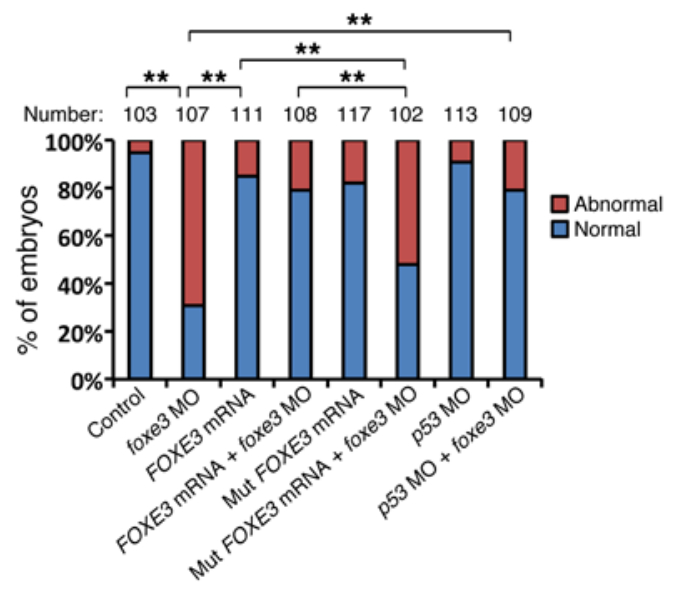

Figure 2. Knockdown of foxe3 disrupts aortic arch development in zebrafish. (A) Representative images of control and foxe3 MO-injected $\mathrm{Tg}(f \mid k 1: E G F P)$ zebrafish at 105 hours post fertilization (hpf). The majority of foxe3 morphants displayed an incomplete or missing aortic arch assembly when compared with control MO-injected embryos. Red letters (AA1-AA6) indicate the location of normal and abnormal aortic arches in control and foxe3 M0-injected zebrafish. Original magnification, $\times 10$. (B) Frequency of aortic arch abnormalities associated with embryos injected with the following: $4 \mathrm{ng}$ scramble MO, foxe3 $\mathrm{MO}$; human FOXE3; WT human mRNA plus foxe3 MO; mutant (Mut) FOXE3 mRNA; mutant FOXE3 mRNA (harboring p.Asp153His); mutant FOXE3 mRNA plus foxe3 MO; $p 53 \mathrm{MO}$; and $p 53 \mathrm{MO}$ plus foxe 3 MO. Aortic arch development was disrupted in $70 \%$ of foxe 3 morphants. Coinjection of the foxe3 MO with human FOXE3 mRNA decreased the percentage of embryos with abnormal arch defects from $70 \%$ to $21 \%(P=0.0003)$. However, coinjection of FOXE3 mRNA harboring the mutation $\mathrm{p}$.Asp153His rescued significantly fewer embryos than did WT mRNA $(52 \%, P=0.001)$. Injection of WT or mutant FOXE3 mRNA alone had no significant effect on aortic arch morphology. Coinjection of the $p 53 \mathrm{MO}$ also rescued foxe3 $\mathrm{MO}$-induced aortic arch development. ${ }^{* *} P<0.01$, by 1-way ANOVA. dent depletion (CADD) score greater than 15, and alter highly conserved amino acids (Supplemental Table 1). FOXE3 encodes a forkhead transcription factor, and 4 of the 7 rare variants (Gly137, Asp153, Asp156, and Arg164) are located in the highly conserved forkhead DNA-binding domain. In contrast, p.Pro112Ser, p.Gly196Ala, p.Pro202Leu, and p.Ser300Gly are predicted to be benign by 6 functional prediction programs and alter amino acids that are not conserved and are thus less likely to cause disease (Supplemental Table 1). The FOXE3 variant p.Asp156Asn has an MAF of 0.029\% in the Exome Aggregation Consortium (ExAC) database (http://exac.broadinstitute.org/), and, therefore, the pathogenic significance of this variant is unclear.

Mutations in FOXE3 have been previously reported to cause a spectrum of eye disorders including Peters anomaly, cataracts, and anterior segment dysgenesis $(10,11)$. Interestingly, FOXE3 mutations causing lens diseases disrupt amino acids in the forkhead domain proximal to the variants associated with FTAAD or outside this domain (Figure 1D). Furthermore, the individuals with TAAD who carry the FOXE3 variants do not have any apparent eye abnormalities. Taken together, these data strongly suggest that unique FOXE3 mutations within the forkhead domain predispose individuals to TAAD.

Knockdown offoxe 3 disrupts aortic arch development in zebrafish. Since FOXE3 has no known role in the aorta, we initially explored its function in aortic development in zebrafish embryos, because the forkhead domain of FOXE3 is highly conserved from humans to zebrafish. Previous studies had shown that foxe 3 is expressed in the lens of the eye and prechordal plate region in zebrafish, and a morpholino (MO) oligomer-induced knockdown of translation disrupted lens morphogenesis $(12,13)$. We injected the same foxe $3 \mathrm{MO}$ into 1-cell-stage $\mathrm{Tg}(f l k 1: E G F P)$ zebrafish embryos, which express EGFP in endothelial cells under the control of the zebrafish $f l k 1$ promoter, to assess the development of the vasculature $(14,15)$. The aortic arches were disrupted in $70 \%$ of foxe 3 morphants, without alterations in the cardiac outflow tract (as illustrated at 105 hours in Figure 2A). Coinjection of the foxe $3 \mathrm{MO}$ with human FOXE3 mRNA decreased the percentage of embryos with an abnormal aortic arch from $70 \%$ to $21 \%(P=0.0003$; Figure $2 \mathrm{~B}$ ), thus confirming the specificity of the vascular defects and cross-species conservation of foxe3. However, coinjection of FOXE3 mRNA harboring the mutation identified in TAA337 (p.Asp153His) rescued significantly fewer embryos compared with WT mRNA (52\%, $P=0.001)$. To examine the potential off-target effects caused by MO toxicity, the foxe $3 \mathrm{MO}$ was coinjected with a p53 MO. Unexpectedly, we found that the p53 MO rescued the aortic arch defects of foxe 3 morphants (Figure 2B). These results raise the possibility that p53 may act downstream of foxe 3 in a pathway that is required for aortic development.

Foxe 3 is critical for ascending aortic development and pressureinduced aortic remodeling in mice. Given the the observation that foxe3 knockdown disrupts aortic development in zebrafish, we sought to characterize the role of Foxe3 in the mouse aorta. Since Foxe 3 mRNA and protein expression could not be detected in adult mouse aortae, we assessed Foxe 3 expression during mouse development. Whole-mount in situ hybridization (ISH) of mouse embryos showed that Foxe3 expression begins at E9.5 in the pharyngeal arches (PAs), the lens placode of the eye, and the caudal forebrain and presumptive midbrain, as previously described (Figure 3A and ref. 16). At E9.75, Foxe3 is expressed in PA1 and PA2, with minimal expression in PA4. By E12.5, Foxe3 is no longer expressed in the PA but is confined to the anterior lens epithelium. The expression pattern of Foxe 3 is similar to that of Pax3, a marker of early NC, which differentiates into SMCs that populate the ascending thoracic aorta $(3,17,18)$. 
A

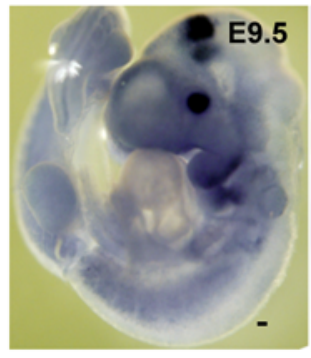

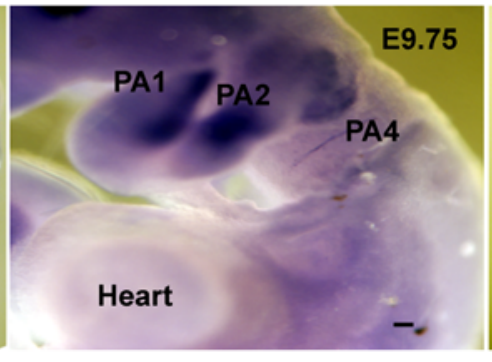

E10.25

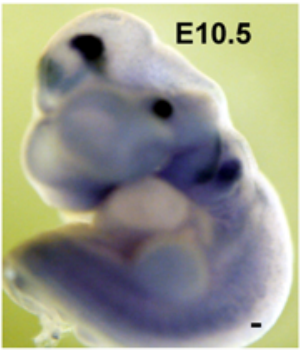

B

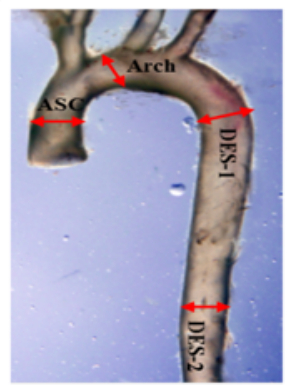

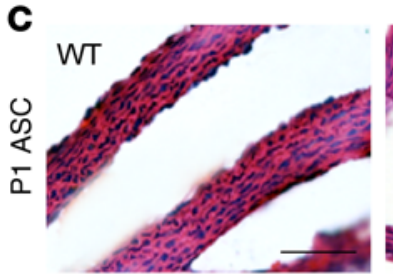
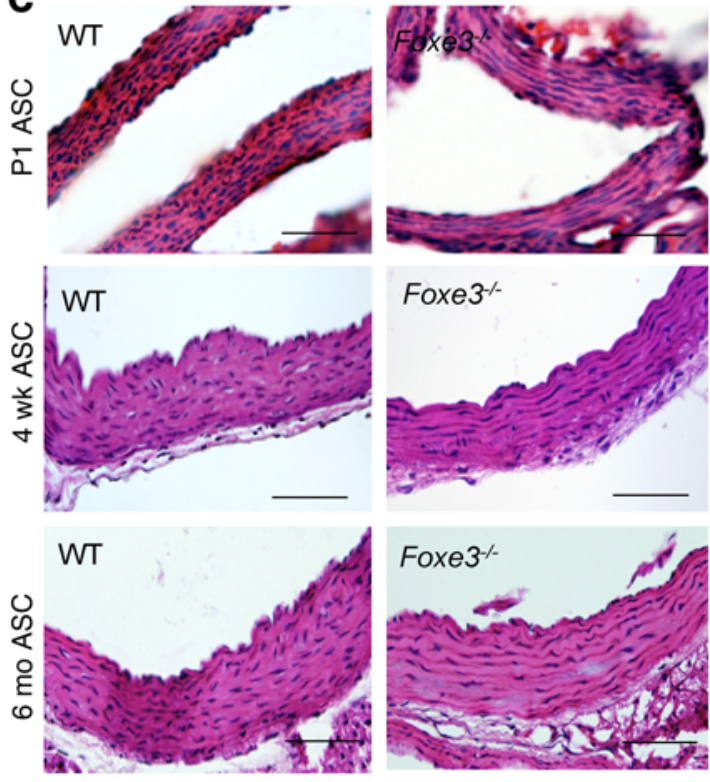
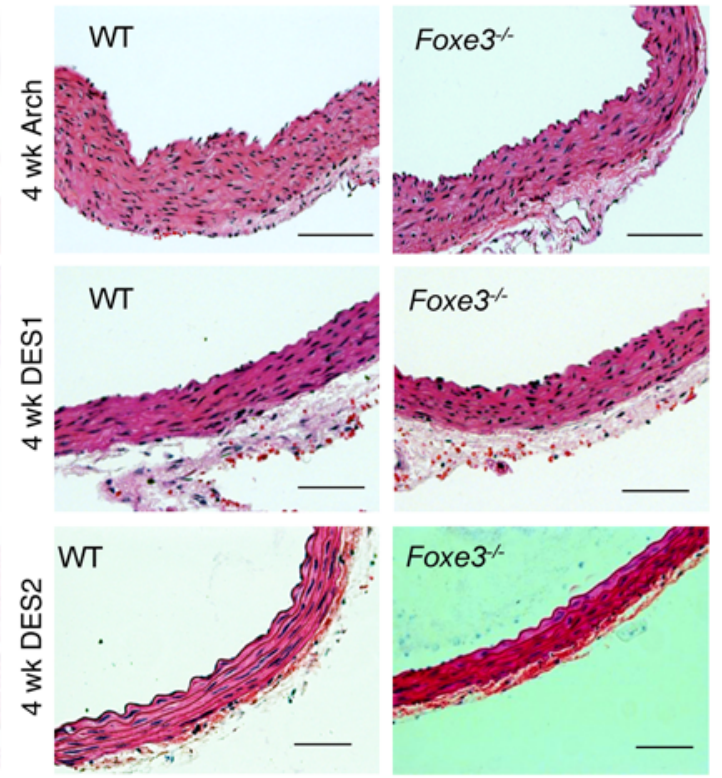

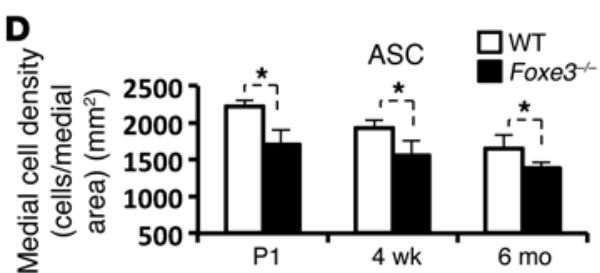

E
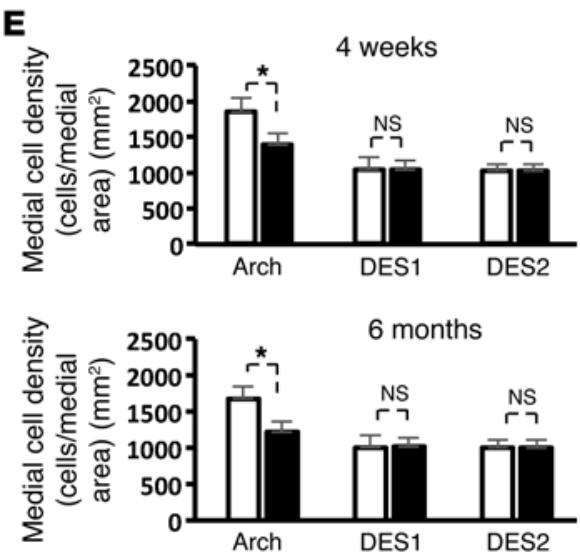

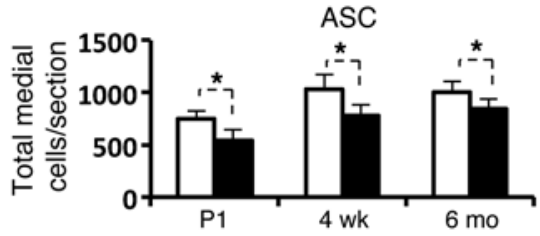

4 weeks
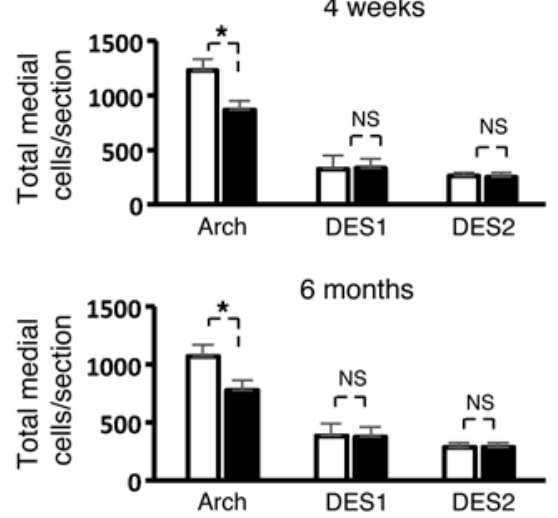

Figure 3. Mice express Foxe 3 in PAs during development, and $\mathrm{Foxe}^{-{ }^{--}}$mice have reduced medial cell density and total numbers of medial cells in the ascending aorta and aortic arch. (A) Expression of the Foxe3 transcription factor. Wholemount ISH of WT mouse embryos (E9.5-E10.5) showed Foxe3 expression in the lens of the eye, in the forebrain/midbrain, and in the PAs; no expression was detected in the heart or its outflow tract. The higher-magnification image shows Foxe3 expression in PA1 and PA2, with weak expression in PA4. (B) Mouse aorta illustrates the regions of the aorta that were analyzed, indicating the ascending aorta, aortic arch, and DES1 and DES2. Original magnification, $\times 100$. (C) Representative $\mathrm{H} \& \mathrm{E}$-stained cross sections of ascending aorta, aortic arch, and descending aortae from male WT and Foxe $^{-/-}$mice at age P1, 4 weeks, and 6 months. (D) Bar graphs show that medial cell density (mean number of medial cell nuclei divided by the total medial area) and total number of medial cells per cross section of the ascending aorta, aortic arch, or descending aorta at day 1, 4 weeks, or 6 months of age were lower in the Foxe $3^{-/-}$mice compared with WT mice. (E) Medial cell density and total number of medial cells were significantly lower in the ascending aortae and aortic arches in Foxe $3^{-1-}$ mice than in WT mice, but there was no statistical significant difference for the descending aorta. $n=5$ per group. Error bars indicate SD from the average. ${ }^{*} P<0.05$, by Student's $t$ test. Scale bars: $100 \mu \mathrm{m}$. ASC, ascending aorta. 
A

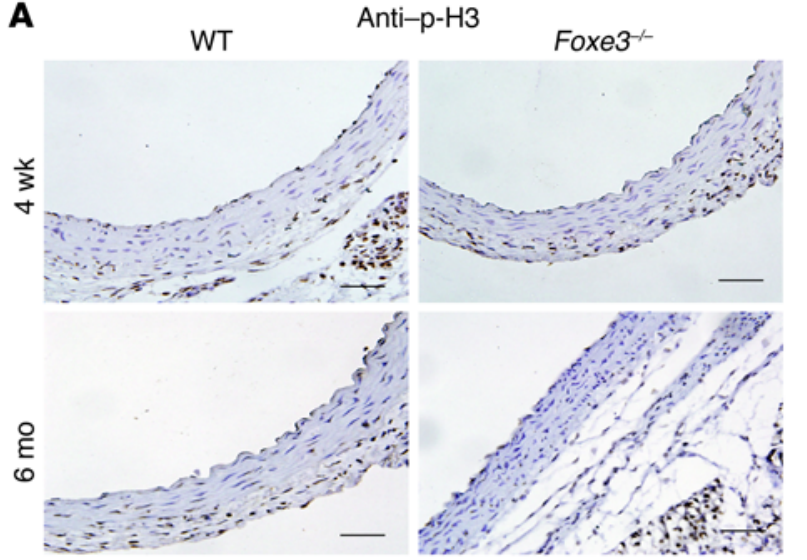

B
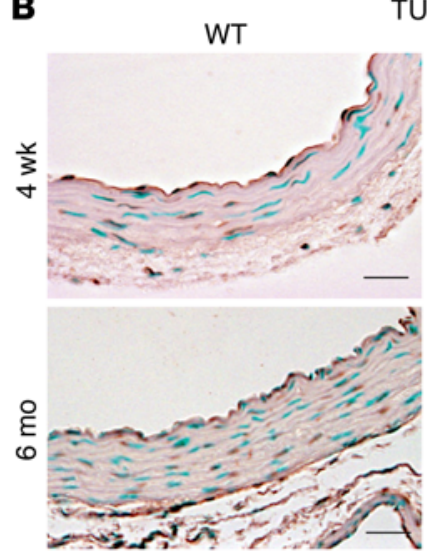

TUNEL
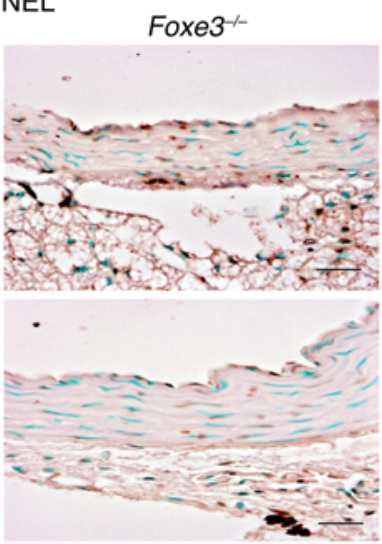

C
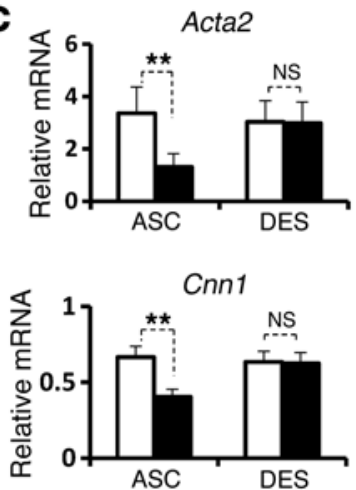

Myh11
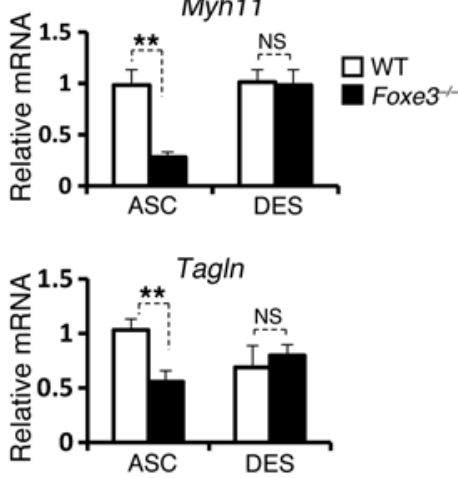

D
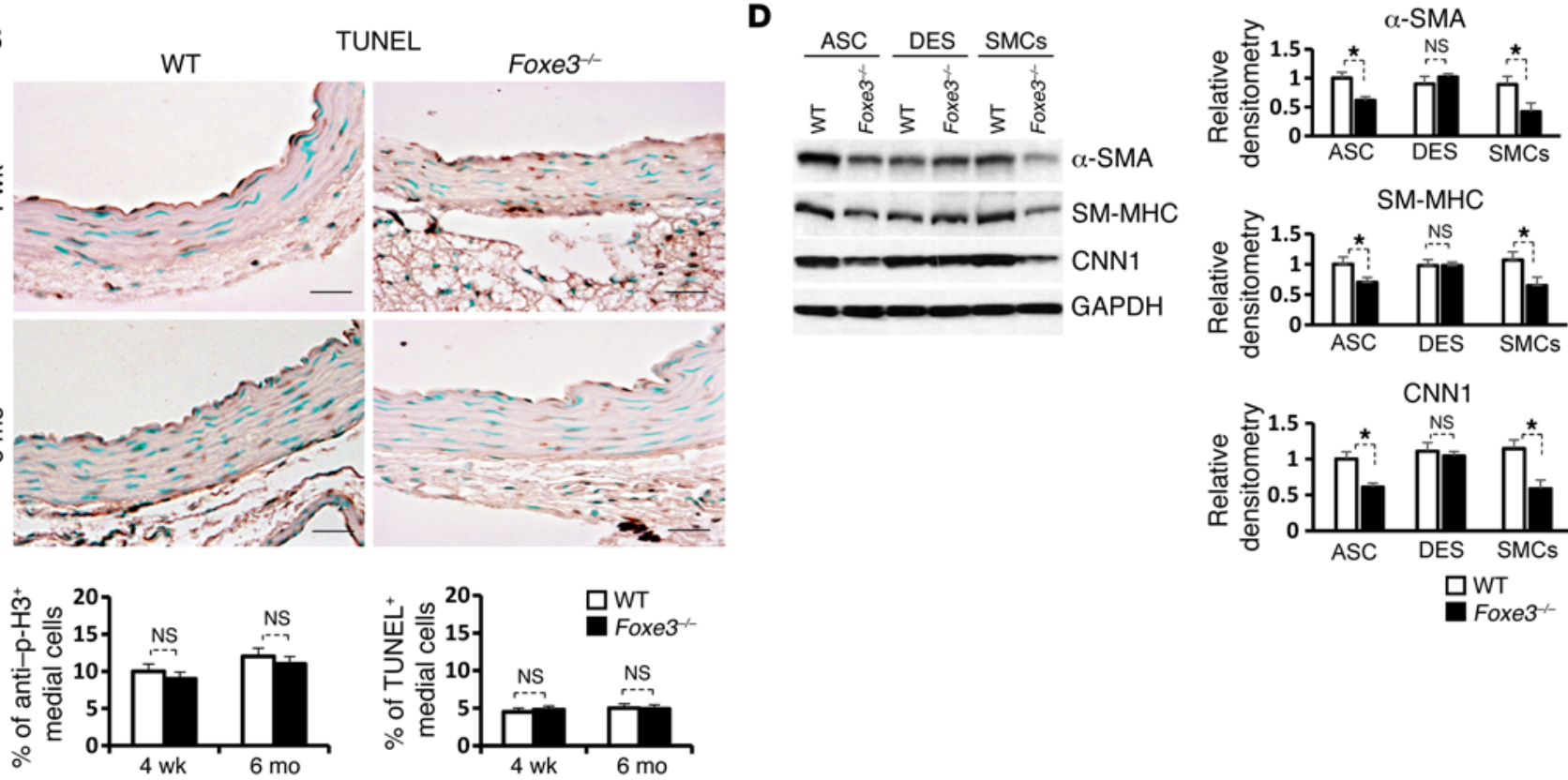

Figure 4. Foxe $3^{-1-}$ ascending aortae have reduced expression of SMC genes encoding contractile protein but show no difference in proliferating or TUNEL-positive cell levels compared with WT controls. (A) Anti-p-H3 staining ( $\mathrm{p}-\mathrm{H} 3$, for proliferative cells) of representative cross sections of ascending aortae from WT and Foxe $3^{-1-}$ mice at 4 weeks and 6 months of age. The cellular proliferation marker showed no differences between WT and Foxe $3^{-1-}$ aortae from mice of the same age. Scale bars: $100 \mu \mathrm{m}$. (B) TUNEL staining in ascending aortic sections from WT and Foxe $3^{-/-}$mice at 4 weeks and 6 months

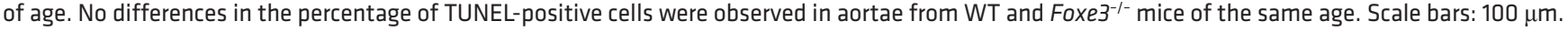
Error bars indicate SD from the average. (C) Real-time qPCR analysis of SMC differentiation marker genes (Acta2, Myh11, Cnn1, and TagIn) using RNA from ascending and descending aortic tissues from WT and Foxe $3^{-/-}$mice (expression levels were normalized to Gapdh). $n=5$ per group. ${ }^{* *} P<0.01$, by Student's $t$ test. (D) Western blots for the SMC contractile proteins $\alpha$-SMA, smooth muscle myosin heavy chain (SM-MHC), and calponin (CNN1) in ascending aortae, thoracic descending aortae, and explanted ascending aortic SMCs from WT and Foxe $3^{-1-}$ mice. Bar graphs show densitometric quantification for $\alpha$-SMA, SM-MHC, and CNN1 in ascending aortae, descending aortae, and SMCs from WT and Foxe $3^{-/-}$mice. Protein levels were significantly lower in the ascending aortae and explanted SMCs from Foxe $3^{-/-}$mice compared with levels detected in WT mice, but there was no difference in levels in the descending aortae. $n=5$ per group. ${ }^{*} P<0.05$, by Student's $t$ test. Error bars indicate SD from the average. DES, descending aorta.

Foxe $^{--}$mice have lens defects but are otherwise indistinguishable from their WT littermates in terms of development, growth, life expectancy, reproduction, and appearance. Foxe3-- aortae have no morphologic abnormalities by both visual inspection and echocardiographic assessment when compared with WT mice aortae (Supplemental Figure 1). At P1, 4 weeks, and 6 months of age, histopathologic analysis of the ascending aortae and aortic arches of Foxe $3^{--}$mice revealed consistently decreased numbers of cells and cell density in the media; no other differences were observed when compared with the WT controls (Figure 3, B-E, and Supple- mental Figure 1, A and C). In contrast, the number and density of medial cells in the proximal and distal thoracic descending aorta (DES1 and DES2, respectively) did not decrease in Foxe3 ${ }^{--}$mice (Figure 3, C and E, and Supplemental Figure 1). There was no difference in the percentage of proliferating cells (assessed by antiphospho-histone H3 [anti-p-H3]) or apoptotic cells (assessed by TUNEL assays) in WT and Foxe $3^{-/}$aortae (assessed at 4 weeks and 6 months; Figure 4, A and B). Quantitative PCR (qPCR) showed that expression of the SMC differentiation markers Acta2, Myh11, Cnn1, and Tagln was also significantly downregulated in Foxe $3^{-1-}$ 

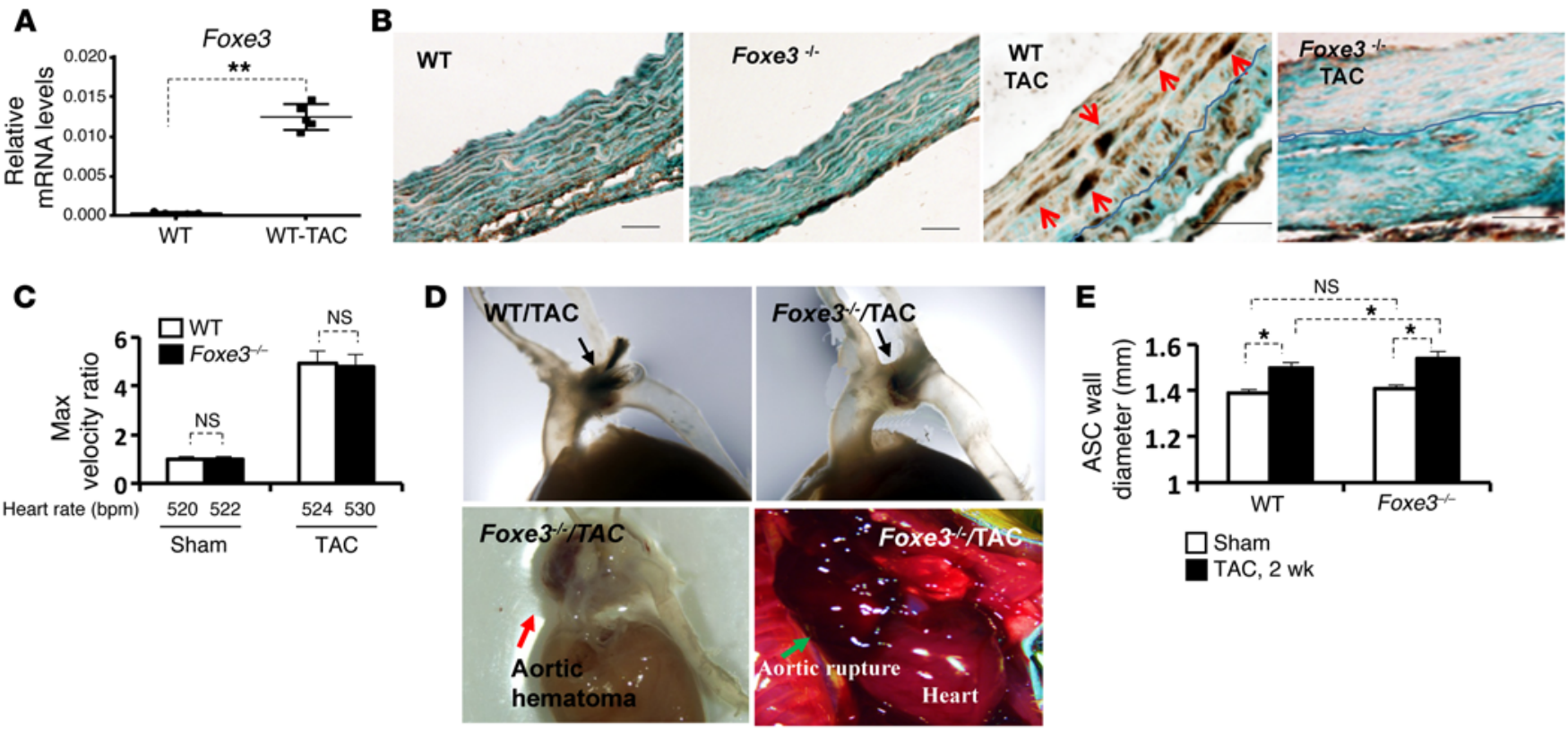

$$
\text { Heart rate }(\mathrm{bpm}) \frac{520522}{\text { Sham }} \quad \frac{524530}{\text { TAC }}
$$

$\mathbf{F}$
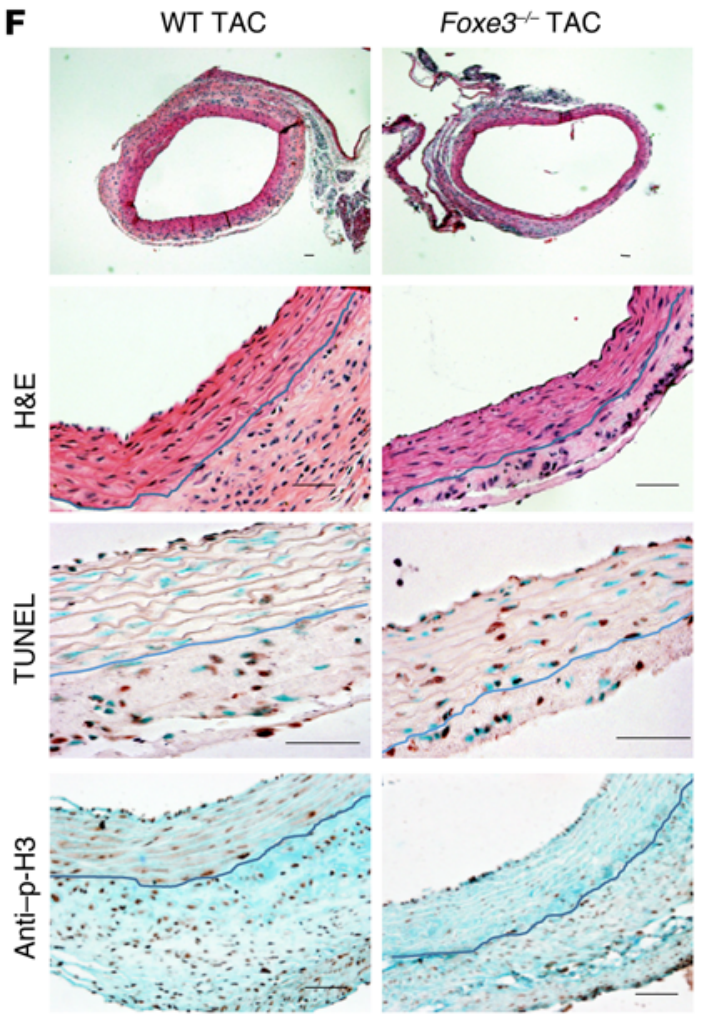
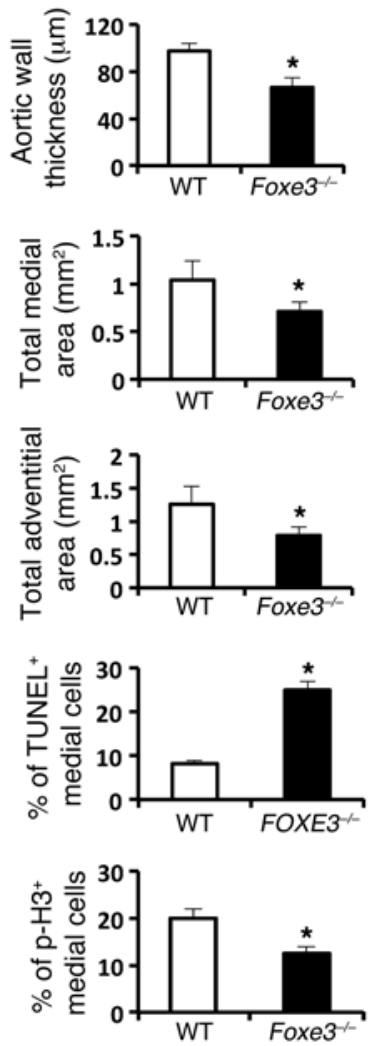

Figure 5. Foxe $3^{-/-}$mice have altered aortic remodeling and rupture of the aorta after TAC. (A) qPCR analysis of Foxe 3 expression in WT ascending aortae 2 weeks after TAC indicated that Foxe3 expression was induced with TAC. $n=5$ per group. ${ }^{* *} P<0.01$, by Student's $t$ test. (B) Cross sections of ascending aortae from mice before and after TAC, immunostained with anti-FOXE3 Ab. Foxe3 staining was not detected in aortic medial layer tissue from WT mice

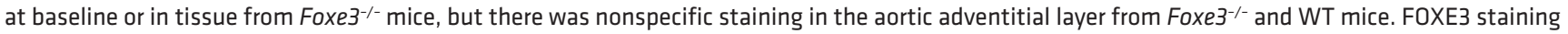
was detected in the medial layer of ascending aortae from WT mice after TAC; adventitial layer staining was also present but may have been nonspecific. (C) Echocardiograms show the maximum (Max) systolic velocities and heart rate (bpm) in ascending aortae after sham operation or TAC. (D) Whole mounts of aortae from mice after TAC; arrows show banding location. Half of the aortae from Foxe $3^{-1-}$ mice were similar to those from WT mice (upper 2 panels), but half of the Foxe $3^{-/-}$mice had complications including aortic hematoma formation (left lower panel, red arrow) or death due to aortic rupture (right lower panel; green arrow). Original magnification, $\times 15$. (E) Echocardiographic assessment of the ascending aortic lumen in WT and Foxe $3^{-/-}$mice after sham operation or TAC. $n=6$ per group. ${ }^{*} P<0.05$, by 1 -way ANOVA. (F) Cross sections of ascending aortae from WT and Foxe $3^{-/-}$mice 2 weeks after TAC with $\mathrm{H} \& \mathrm{E}$, TUNEL, and anti-p-H3 staining. Blue line shows the boundary between the media and adventitia. Bar graphs show the quantification of aortic wall thickness, total medial area, total adventitial area, and the percentage of TUNEL-positive and anti-p-H3-positive medial cells. $n=5$ per group.

${ }^{*} P<0.05$, by Student's $t$ test. 
ascending aortic tissues when compared with WT aortae, and this was confirmed by Western blot analysis of ascending aortic lysates and explanted SMCs (Figure 4, C and D). However, expression levels of the same SMC marker genes were not significantly different in the descending thoracic aortae of WT and mutant animals (Figure 4, C and D). These histologic changes persisted in aged Foxe $3^{-1-}$ mice (1.6 years old), but we observed no other pathologic changes in comparison with age-matched WT animals (Supplemental Figure 2). Therefore, deficiency of Foxe3 during development leads to specific reductions in the number, density, and differentiation status of SMCs in the ascending aorta and aortic arch.

To determine whether Foxe $3^{-/-}$aortae were more sensitive to risk factors for aortic disease, we used the established model of transverse aortic constriction (TAC) to increase biomechanical force on the ascending aorta $(19,20)$. After TAC, Foxe3 expression was significantly induced in WT mice, and immunostaining detected FOXE3 protein in the nuclei of medial cells in the ascending aorta (Figure 5, A and B). Doppler measurements of aortic flow velocity were not significantly different between $\mathrm{Foxe}^{-/-}$and WT mice after TAC (Figure 5C). However, 4 of 14 Foxe $^{-/-}$animals died between 4 and 11 days after TAC, while there was no mortality among the WT controls. At necropsy, we found hemothoraces indicative of aortic rupture in $\mathrm{Foxe}^{-/-}$animals but not in the controls (Figure 5D and Supplemental Figure 3A). Furthermore, 3 surviving $\mathrm{Foxe}^{-/-}$animals had blood in the proximal ascending aortic wall after 2 weeks of TAC (Figure 5D). Histopathology of the hemorrhagic aorta demonstrated medial degradation, with inflammatory infiltrates and thrombus, consistent with intramural hematomas (Supplemental Figure 3A). Thus, 50\% of Foxe $3^{-/-}$(7 of 14) and none of the WT control ( 0 of 13) mice developed TAC-induced aortic complications (Figure 5D and Supplemental Figure 3A; $P<0.01)$. In addition, the ascending aortae of the surviving Foxe $3^{-1-}$ mice were significantly dilated in comparison with those of the WT controls (Figure 5E and Supplemental Figure 3B; $P<0.05$ ).

TAC induces thickening of the media and adventitia of the ascending aortic wall in WT mice (20), but the Foxe3 ${ }^{-/-}$aortae showed significantly less thickening, with reduced medial and adventitial areas, compared with WT aortae 2 weeks after TAC $(P<0.05$; Figure $5 \mathrm{~F}$ and Supplemental Figure $3 \mathrm{C})$. TAC also induces an inflammatory reaction characterized by increased expression of matrix metalloproteinases (Mmp2, Mmp9) and inflammatory mediators (Il6) and infiltration of macrophages in the adventitia (20). Induction of Mmp2, Mmp9, and Il6 expression as well as adventitial macrophage infiltration (positively stained antiMOMA2 or anti-Mac2 cells) after TAC were significantly attenuated in Foxe $3^{-/-}$aortae (Supplemental Figure 4, A and B; $P<0.01$ ). Finally, expression of SMC markers, including Acta2, Myh11, and Cnn1, was significantly induced with TAC in WT aortae, while it was correspondingly reduced in Foxe $3^{-/-}$aortae (Supplemental Figure 4C). These observations reveal that FOXE3 deficiency impairs ascending aortic remodeling in response to mechanical stress.

To investigate how Foxe3 may affect SMC survival, markers of apoptosis and proliferation were measured in the ascending aortae of $\mathrm{Foxe}^{-/-}$and WT mice. Two weeks after TAC, the percentage of TUNEL-positive cells was significantly increased in the aortic media of Foxe $3^{--}$animals compared with that seen in WT controls (Figure 5F; $P<0.05$ ). Conversely, the percentage of anti-p-H3-pos- itive SMC nuclei in the media of Foxe $3^{-/-}$aortae was significantly decreased (Figure 5F; $P<0.05$ ). Thus, FOXE3 deficiency attenuates stress-induced aortic remodeling, as indicated by relative thinning of the aortic wall and increased SMC apoptosis after TAC.

Inhibition of 553 rescues aortic developmental defects and aortic remodeling in response to increased biomechanical forces in Foxe $3^{-1-}$ mice. Our zebrafish data suggested that aortic defects induced by loss of foxe 3 are p53 dependent (Figure 2). Therefore, we sought to determine whether aberrant TAC-induced aortic remodeling and increased SMC apoptosis in the Foxe $3^{-/}$mice were also dependent on p53. We initially determined that Foxe $3^{-1-}$ SMCs explanted from ascending aortae were significantly more sensitive to staurosporine-induced apoptosis than were WT SMCs, as assessed by the percentage of SMCs positive for annexin V (Figure 6A). When the experiment was performed with pifthirin- $\alpha$ (PFT), a pharmacological p53 inhibitor (21), the percentage of annexin V-positive Foxe $3^{-1-}$ SMCs decreased to WT levels (Figure 6A). We then sought to determine whether $\mathrm{p} 53$ inhibition could prevent aortic apoptosis in vivo by injecting PFT $(2.2 \mathrm{mg} / \mathrm{kg})$ i.p. into WT and Foxe3-mice 24 hours before TAC and every 48 hours after TAC (21). PFT significantly reduced the incidence of aortic rupture and intramural hematomas in Foxe $3^{-/-}$mice that underwent TAC (50\% to 17\%, $P<0.05)$. After PFT treatment, the mean diameter of the ascending aorta and the percentage of TUNEL-positive cells in the aortic media were also normalized to WT levels in surviving Foxe $3^{-/-}$animals ( $P<0.05$, Figure 6B, echocardiogram and Figure 6C).

To determine whether a constitutive absence of $\mathrm{p} 53$ could rescue aortic developmental defects in $\mathrm{Foxe}^{-/-}$mice, we crossed Foxe $3^{-/-}$mice with $p 53$-deficient $\left(p 53^{-/-}\right)$mice to generate Foxe $3^{-/-}$ and $p 53^{-/-}$double-KO (Foxe $3^{-/-} p 53^{-/-}$) animals. These mutant mice were live born at expected Mendelian ratios and had no apparent congenital abnormalities. In 4-week-old Foxe $3^{-/-} p 53^{-/-}$ ascending aortae, we observed that the total number of medial cells and cell densities were effectively restored to WT levels (Figure 6D). The attenuated expression of SMC differentiation markers that we had observed in Foxe $3^{-/-}$ascending aortae was not significantly improved by $\mathrm{p} 53$ deficiency (Figure 6E). We further investigated whether loss of p53 would rescue the aberrant pressure-induced aortic remodeling seen in Foxe $3^{-/-}$animals. No aortic ruptures or aortic aneurysms or dissections occurred with TAC in the Foxe $3^{-/-} p 53^{-/-}$mice. Echocardiography confirmed that the diameters of the ascending aortae from Foxe $3^{-/-} p 53^{---}$mice 2 weeks after TAC were similar to those in ascending aortae from WT mice (Figure 7B). No aortic enlargement or histologic or pathologic changes were identified in $p 53^{---}$ascending aortae compared with WT aortae at baseline (Supplemental Figure 5). Loss of p53 completely restored TAC-induced Foxe $3^{-/-} p 53^{-/-}$aortic remodeling to the extent observed in WT mice, with increases in medial and adventitial areas that were indistinguishable from those observed in WT aortae (Figure 7, A and C). Similarly, deletion of $p 53$ rescued TAC-induced aortic SMC apoptosis in Foxe $3^{-1-}$ $p 53^{-/}$ascending aortae to levels indistinguishable from those in WT aortae (Figure 7, TUNEL assay). Finally, Foxe $3^{-/-} p 53^{-/-}$aortae showed an increase in the number of p-H3-positive cells similar to that seen in WT aortae (Figure 7, anti-p-H3). Although loss of p53 effectively rescued ascending aorta medial cell density in $\mathrm{Foxe}^{-1-}$ mice, the lens defects in $\mathrm{Foxe}^{-/-}$mice were not rescued 

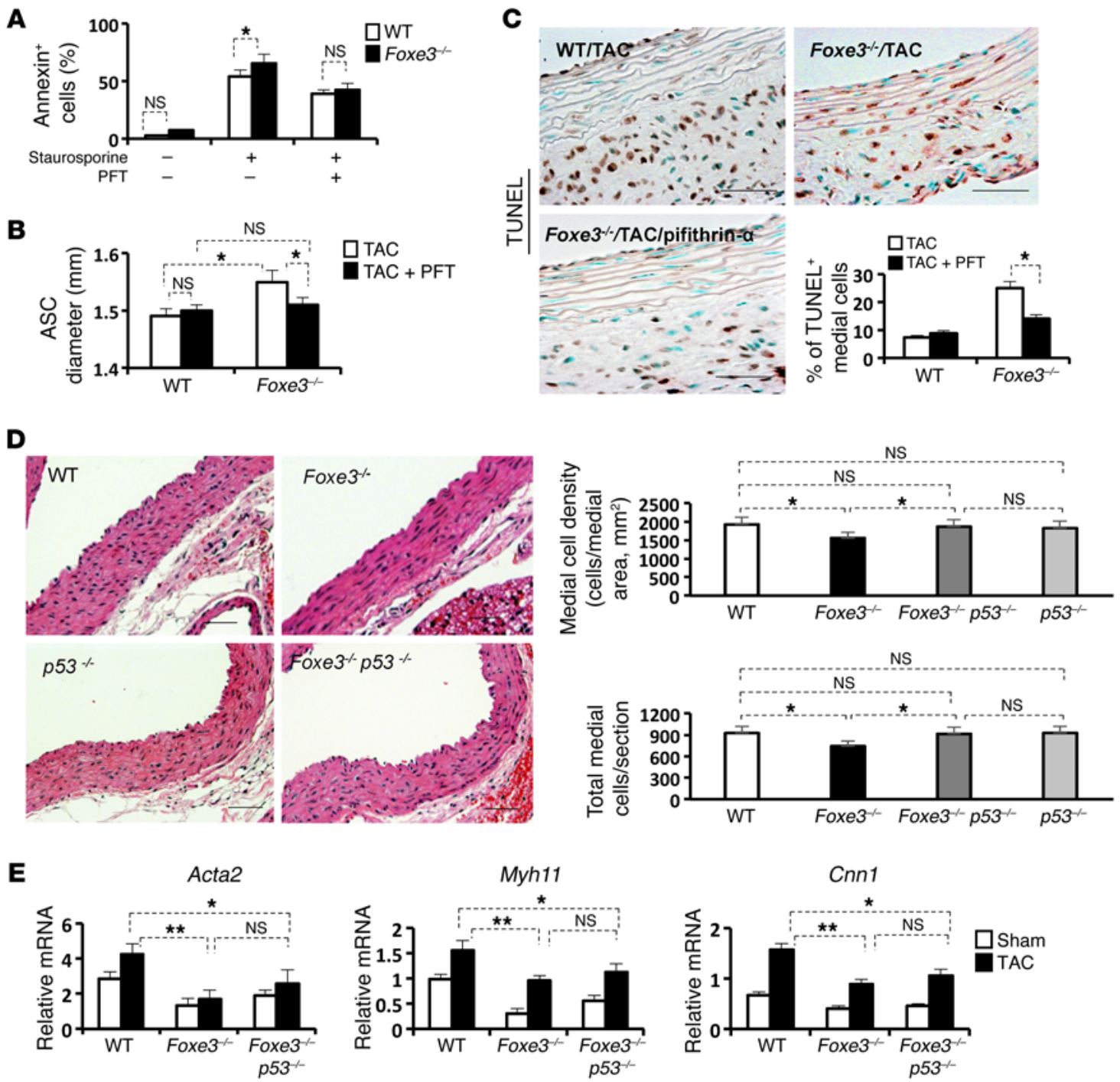

Figure 6. Aortic developmental defects and aberrant aortic remodeling with TAC in Foxe $3^{-/-}$mice is rescued by blocking p53. (A) Annexin $\mathrm{V}$ assay by flow cytometric analysis. After a 24-hour exposure to staurosporine, the percentage of annexin V-positive cells was significantly greater in Foxe $3^{-/-}$SMCs compared with that seen in WT SMCs. Inhibition of p53 with PFT significantly decreased the percentage of annexin V-positive Foxe $3^{-1-}$ SMCs to the level detected in WT SMCs. ${ }^{*} P<0.05$, by Student's $t$ test. (B) Echocardiographic analysis of the ascending aortic diameter shows that PFT treatment rescued the increased aortic diameter with TAC in Foxe3 $3^{-/-}$mice. $n=5$ per group. ${ }^{*} P<0.05$, by 1 -way ANOVA. (C) TUNEL staining in ascending aortic sections from WT and Foxe $3^{-1-}$ mice with TAC and PFT treatment. Bar graphs show that the proportion of TUNEL-positive medial cells in the Foxe $3^{-1-}$ mice decreased with PFT treatment. $n=5$ per group. ${ }^{*} P<0.05$, by Student's $t$ test. Original magnification, $\times 400$. (D) Representative images of H\&E-stained cross sections of ascending aortae from 4-week-old male WT, Foxe $3^{-/-}, p 53^{-/}$, and Foxe $3^{-/-} p 53^{-/-}$mice. Bar graphs show their medial cell densities (mean number of medial cell nuclei divided by the total medial area) and the total number of medial cells per cross section at the ascending aorta. Deletion of $p 53$ effectively restored medial cell density and cell numbers in the ascending aortic media of Foxe $3^{-/-}$mice and eliminated the differences among Foxe $3^{-1-} p 53^{-1-}, p 53^{-1 /}$, and WT mice of the same age. $n=6$ per group. ${ }^{*} P<0.05$, by 1 -way ANOVA. Original magnification, $\times 200$. (E) qPCR analysis of SMC-specific gene (Acta2, Myh11, and Cnn1) expression in ascending aortic tissues from WT, Foxe $3^{-1-}$, and Foxe $3^{-1-} p 53^{-1-}$ mice. Gene expression levels were normalized to Gapdh. $n=5$ per group. ${ }^{*} P<0.05$ and ${ }^{*} P<0.01$, by 1 -way ANOVA.

with $p 53$ deficiency (Supplemental Figure 6). These data suggest that FOXE3 and p53 may interact to regulate SMC survival during development and in response to increased aortic stress in adult animals.

To determine how 553 may drive SMC loss with FOXE3 deficiency, we investigated the role of CDKN1A, a known transcriptional target of p53 that encodes the cyclin-dependent kinase inhibitor p21/WAF $(22,23)$. Although CDKN1A is not expressed in the adult aorta, we found that Cdkn1a expression was induced in mice 2 weeks after TAC, and its expression was significantly greater in the TAC aortae of Foxe $3^{-/}$mice when compared with WT mice aortae (Figure 8A; $P<0.01$ ). Since knockdown of $p 53$ expression effectively rescued the aortic developmental defects associated with loss of foxe 3 expression in zebrafish and to determine whether CDKN1A acts downstream of p53, we coinjected a Cdkn1a MO with the foxe $3 \mathrm{MO}$. We found that knockdown of $C d k n 1 a$ expression also rescued the aortic arch defect of foxe 3 MO-injected zebrafish (Figure 8B; $P<0.01$ ). 
A
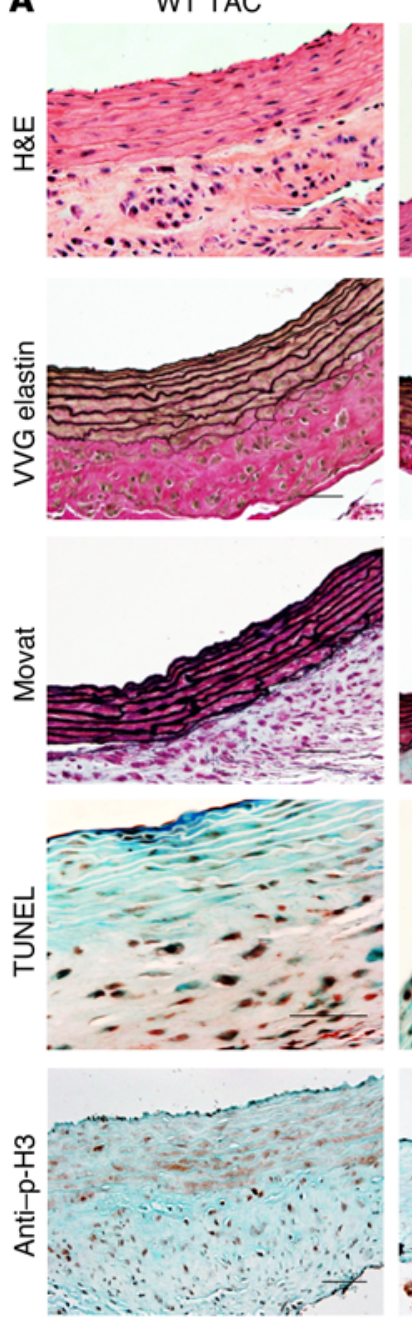

Foxe3 $/$ p53 $/$ TAC
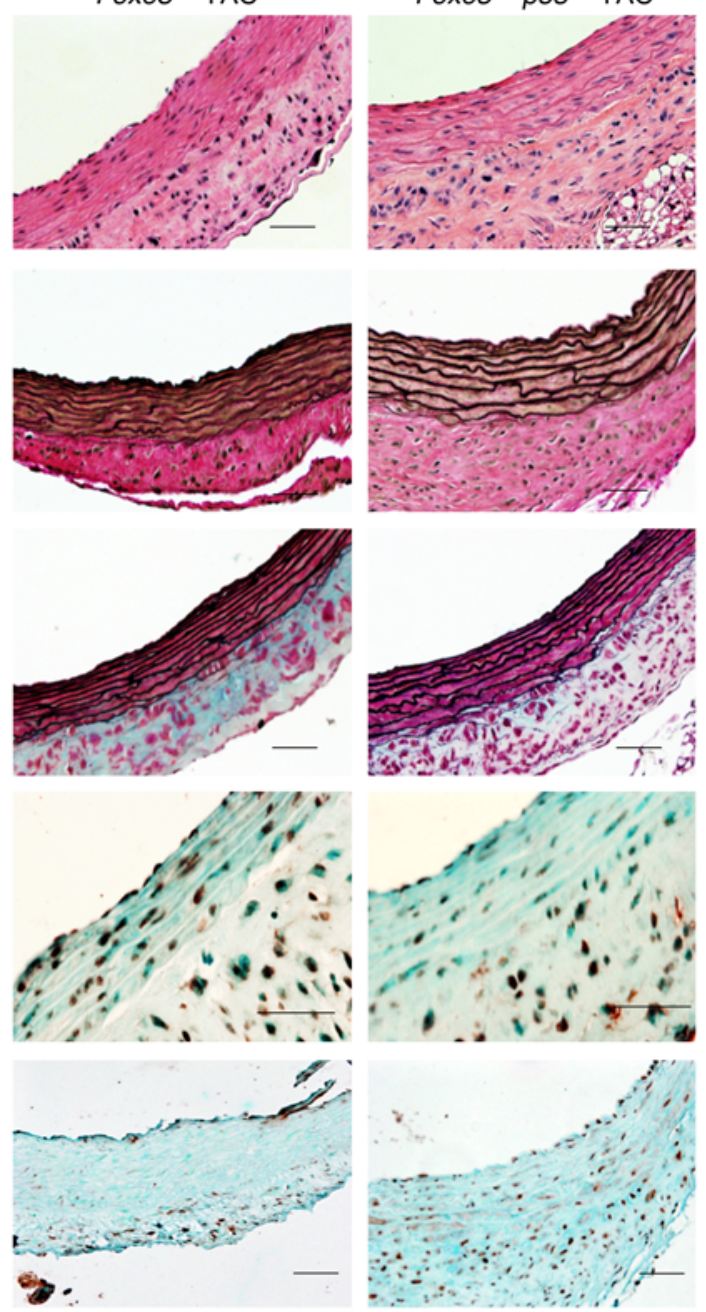

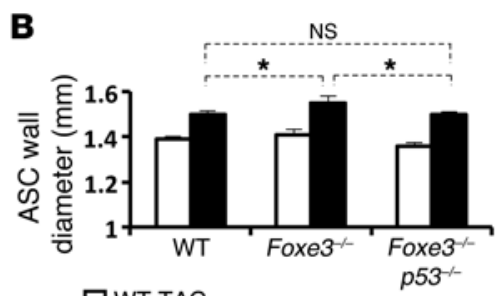

DWT TAC

Foxe3 ${ }^{-T A C}$

C
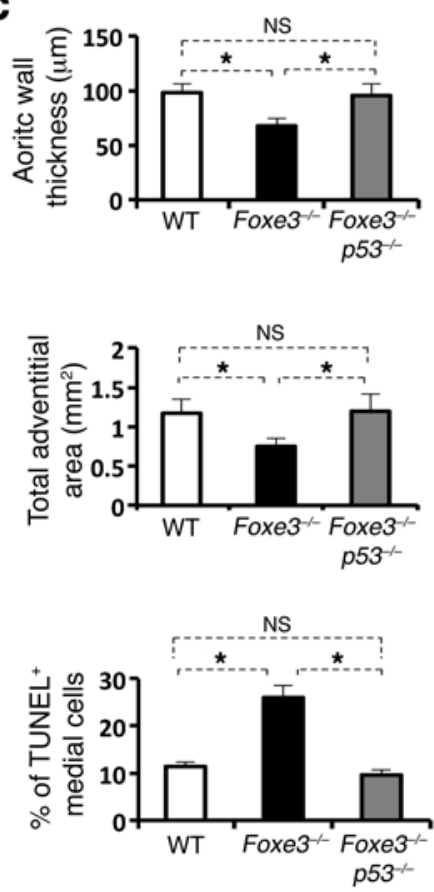
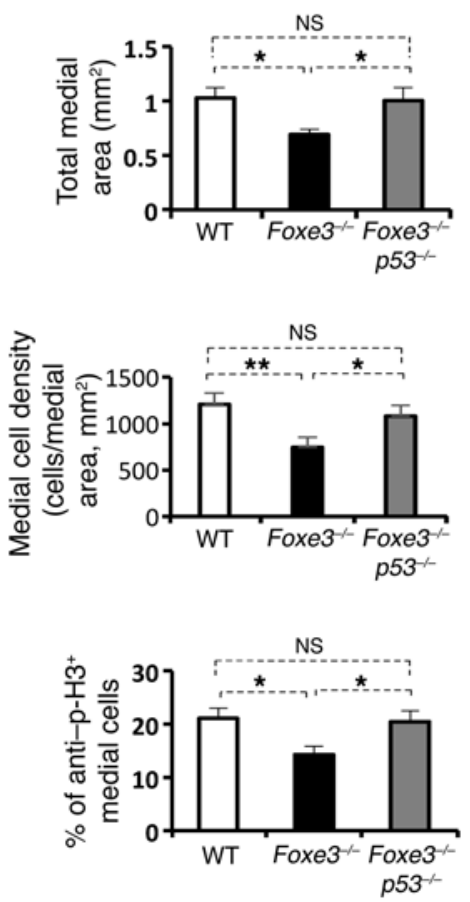

Figure 7. Loss of p53 rescues TAC-induced aortic rupture and SMC apoptosis in Foxe3-deficient aortae. (A) Representative images of ascending aortic cross sections from WT, Foxe $3^{-/-}$, and Foxe $3^{-1-} p 53^{-1-}$ mice 2 weeks after TAC. Cross sections of ascending aortae were stained with $\mathrm{H} \& \mathrm{E}$, VVG elastin, Movat pentachrome, TUNEL, and anti-p-H3. Scale bars: $100 \mu \mathrm{m}$. (B) Echocardiographic analysis of the aortic diameter of ascending aortae from WT, Foxe3 ${ }^{-/-}$, and Foxe3 $^{-/-}$p53 5 -/- mice 2 weeks after TAC. The diameters of the ascending aortae from Foxe $3^{-/-} p 53^{-/-}$mice 2 weeks after TAC were similar to diameters of aortae from WT mice. $n=5$ per group. ${ }^{*} P<0.05$, by 1 -way ANOVA. (C) Bar graphs show the quantification of aortic wall thickness, total medial area, total adventitial area, medial cell density (mean number of medial cell nuclei divided by medial area $\left[\mathrm{mm}^{2}\right]$ ), percentage of TUNEL-positive aortic medial cells, and percentage of medial cells stained with anti-p-H3 Abs. p53 deficiency rescued TAC-induced aberrant aortic remodeling in Foxe $3^{-/-}$mice to an extent indistinguishable from that observed in WT mice, as shown by the increased medial and adventitial areas and number of TUNEL-positive medial cells as well as the increased number of $\mathrm{p}$-H3-positive medial cells. $n=5$ per group. ${ }^{*} P<0.05$ and ${ }^{*} P<0.01$, by 1 -way ANOVA. Error bars indicate SD from the average. 
A
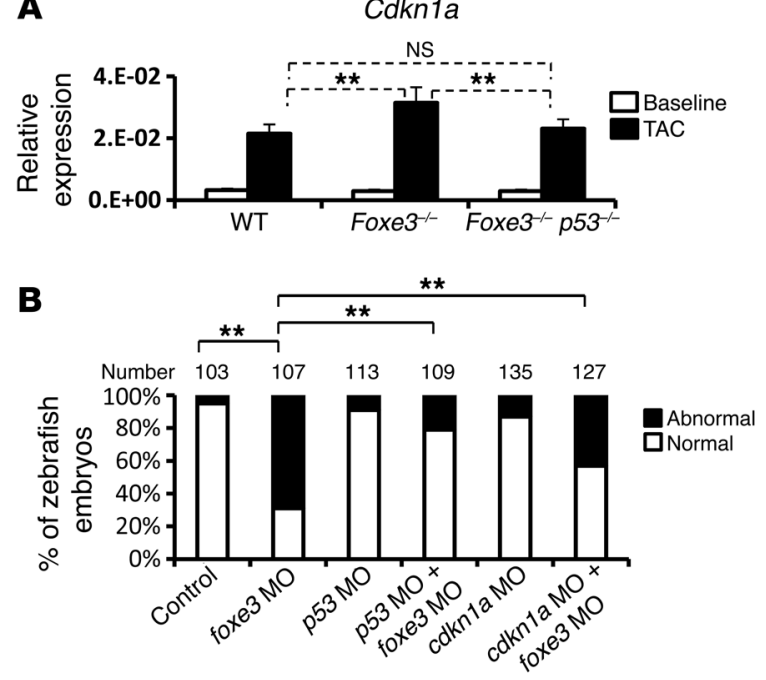

Figure 8. Loss of $p 53$ rescues SMC loss with Foxe3 deficiency through Cdkn1a. (A) qPCR analysis of Cdkn1a mRNA expression in ascending aortae from WT, Foxe $3^{-/}$, and Foxe $3^{-/-} p 53^{-/-}$mice at baseline and 2 weeks after TAC. Cdkn1a was significantly induced with TAC, and expression levels were significantly higher in the TAC aortae from Foxe $3^{-/-}$mice when compared with levels in aortae from WT mice. Deletion of $p 53$ significantly restored Cdkn1a expression levels to those detected in WT aortae. $n=5$ per group. ${ }^{*} P<0.01$, by 1 -way ANOVA. (B) Quantification of aortic arch abnormalities associated with embryos injected with the control MO, foxe3 MO, p53 MO, or cdkna1 MO. Knockdown of foxe3 with the foxe3 MO disrupted development of the aortic arches in zebrafish. Coinjection of the foxez MO with the $p 53 \mathrm{MO}$ significantly rescued the aortic defect. Injection of the cdkna1 $\mathrm{MO}$ also partially rescued foxe $3 \mathrm{MO}$-induced abnormal aortic arch development in zebrafish. ${ }^{*} P<0.01$, by 1-way ANOVA.

\section{Discussion}

We identified dominant FOXE3 mutations in patients with TAADs by a combined approach of whole-exome and Sanger sequencing of DNA from families with multiple members with thoracic aortic disease. The missense mutations identified specifically alter amino acids at the C-terminal end of the forkhead DNA-binding domain. In contrast, dominant FOXE3 mutations that cause ocular conditions are located at the N-terminal end of the forkhead domain or outside this domain $(24,25)$. Additionally, the homozygous dysgenic lens $(d y l)$ mouse has 2 Foxe 3 missense mutations, F93L and F98S, both of which fall at the N-terminal end of the forkhead domain (the homologous amino acids in the FOXE3 are F100 and F105) (26). The distinct location of the FOXE3 mutations causing these 2 phenotypes implicate different effects of these mutations on transcriptional function. Analyses of FOXC1 mutations, which cause a spectrum of ocular defects characterized by disruption of the iris, have found that missense mutations can differentially affect the forkhead transcription factor's stability, DNA binding, or transactivation of gene expression (27). It is also possible that mutations in different regions of the DNA-binding domain disrupt the binding or transactivation of different genes. Although mutations in a single forkhead transcription factor can cause pleiotropic developmental defects and adult-onset diseases (28), there are no other examples in which mutations in different regions of a forkhead transcription factor cause different phenotypes.
The data presented here suggest that Foxe 3 is critical for populating the ascending aorta with differentiated SMCs during development. Interestingly, Foxe3 plays a similar role in the eye; deficiency of Foxe 3 in mice causes reduced proliferation of the anterior lens epithelium, and the differentiating lens fiber cells do not mature properly (29). The data presented here indicate that Foxe3 deficiency limits SMC proliferation or survival and decreases differentiation as the ascending aorta and arch are populated during development. SMCs arise from multiple embryonic origins. The ascending aorta and aortic arch SMCs are derived from the NC, whereas SMCs in the descending thoracic aorta are derived from the paraxial mesoderm (3). Foxe3 expression was present in the PAs during development, which correlates with our finding that only NC-lineage SMCs in the ascending aorta and aortic arch were underpopulated and the descending thoracic aortic SMCs were not. Deficiency of p53 rescued the decreased SMC density and total SMC numbers in the ascending aorta but did not rescue the decreased differentiation of these cells. Previous studies have established a role of p53 in SMC proliferation and survival; loss of $p 53$ drives excessive SMC proliferation, and overexpression of $p 53$ leads to premature SMC senescence (30). Thus, our data indicate that FOXE3 limits p53 activity in NC-derived SMCs during development so that the ascending aorta and aortic arch are sufficiently populated with SMCs.

Our data also indicate that Foxe3 expression is induced with increased biomechanical forces in ascending aortic SMCs and drives antiapoptotic/prosurvival pathways in these cells. Although low levels of SMC apoptosis are evident in thoracic aortic aneurysms, a role of SMC apoptosis in disease initiation or progression has not been established (30). Studies using mouse models engineered with both inducible and chronic vascular SMC-specific apoptosis have demonstrated that SMC apoptosis can alter aortic pathology, specifically increasing both breaks in the elastin lamellae and proteoglycan accumulation in the aortic media (31). Although current data suggest excessive TGF- $\beta$ signaling as the cause of aortic disease in the Marfan mouse model ( $F b n 1^{10396 /+}$ ) (32), these mice show evidence of aortic SMC apoptosis in the postnatal period. Furthermore, a pan-caspase inhibitor attenuates aneurysm growth in these mice (33). Identification of Foxe3 deficiency as a cause of stress-induced apoptosis of aortic SMCs leading to aortic ruptures provides additional support for a role of SMC apoptosis in thoracic aortic disease and, specifically, a role of SMC apoptosis in thoracic aortic ruptures.

It is notable that both p53 deficiency and PFT treatment rescued the increase in SMC apoptosis and deaths due to aortic rupture in Foxe $3^{-1-}$ mice. Although p53 deficiency rescued the decreased number of SMCs in the ascending aorta, treatment with PFT was sufficient to attenuate SMC apoptosis and aortic rupture in Foxe $^{-/-}$mice, despite the decreased numbers and dedifferentiation of SMCs in the ascending aorta. PFT was initially identified as a compound capable of inhibiting p53-mediated apoptosis (34). More recently, it has been reported that PFT reduces apoptosis in a human colon cancer cell line (HCT116 cells) in the absence of p53, acting through mitochondria but upstream of caspase 9 activation (35). Aortic dissections and ruptures are what lead to premature death from thoracic disease, and these data suggest that therapeutic manipulation of SMC prosurvival pathways has the potential to decrease the risk for these deadly complications. 


\section{Methods}

Family recruitment and characterization. Families with 2 or more members affected with thoracic aortic diseases were enrolled in the study (36). Phenotypic characterization of vascular diseases was previously described (36). Blood or saliva samples were obtained from affected individuals and family members. Medical records, including imaging studies of the aorta, surgical reports, hospital records, death certificates, and physicians' notes were reviewed. Phenotypic features beyond the vascular system were assessed in FOXE3 mutation carriers who were examined by clinical geneticists. Familial thoracic aortic disease probands were from a Houston cohort $(n=297)$, along with probands from the National Registry of Genetically Triggered Thoracic Aortic Aneurysms and Cardiovascular Conditions (GenTAC) cohort $(n=58)$. The ethnic and racial backgrounds of the 355 affected probands were as follows: 91\% European American, 4\% African American, $3 \%$ Hispanic, $1 \%$ Asian, and $1 \%$ other ethnicity.

French probands and families $(n=41)$ were recruited through the National Reference Center for Marfan Syndrome and Related Disorders (Centre National de Référence pour le syndrome de Marfan et apparentés) and through related centers throughout France. All of these patients were of European descent and were screened for diagnosis of a possible syndromic cause of TAADs through a multidisciplinary clinic, including systematic slit-lamp examination and extensive imaging.

Exome sequencing. Genomic DNA was extracted from peripheral blood lymphocytes using standard protocols. Five micrograms of DNA from 2 affected individuals in family TAA337 (coefficient of relationship $=1 / 16$ ) was used for construction of the shotgun sequencing library as described previously using adapters for paired-end sequencing (37). Exome sequences were captured by SeqCap EZ Exome Probes, version 1.0 (Roche) and recovered according to the manufacturer's directions. Enriched libraries were then sequenced on an Illumina Genome Analyzer IIx following the manufacturer's protocols.

Reads were mapped to the reference human genome (UCSC hg19) with the Burrows-Wheeler Aligner (BWA) (SourceForge) and variants called using SAMtools (37). Insertion-deletion (indel) variants affecting the coding sequence were identified after a Smith-Waterman realignment of the BWA calls. Single nucleotide variants (SNVs) and indels were filtered to greater than 8 times and quality to greater than 30. Annotation of variants was performed using the SeattleSeq server (http://snp.gs.washington.edu/SeattleSeqAnnotation141/). Approximately 16,800 heterozygous variants were identified in each individual. We further filtered out synonymous variants, variants that were located outside splicing sites or coding regions, or variants that had an MAF of more than $0.05 \%$ in the European American cohort in the NHLBI ESP. By this filtering strategy, 213 and 199 variants, respectively, were identified, and 13 of these variants were heterozygous in both affected individuals. These variants were considered candidate mutations causing thoracic aortic disease. The exome sequencing data for this family have been deposited in the dbGaP database (dataset name: ESP_Family_Studies_Aortic_Disease_Subject; dataset accession: pht003123.v1.p1).

DNA sequencing. A Sanger DNA sequencing assay was performed to validate exome sequencing results and test the segregation of variants with TAAD in families (37). FOXE3 mutations were reported on the basis of the NCBI's RefSeq code NM_012186.2.

In vivo studies in zebrafish. $\mathrm{Tg}(f l k 1: E G F P)$ zebrafish were maintained at $28.5^{\circ} \mathrm{C}$ under continuous water flow and filtration, with automatic control for a 14-hour light/10-hour dark cycle. The night before injection, male and female fish were placed into a 1-liter tank containing a fish mating cage with an inner mesh and divider. Zebrafish embryos were obtained from natural spawning by removing the divider and stimulating with light. The embryos were kept at $28.5^{\circ} \mathrm{C}$ before and after microinjection. The foxe $3 \mathrm{MO}$ was injected into the yolk of 1- to 2-cell-stage embryos at a dosage of $4 \mathrm{ng}$ per embryo. The foxe 3 MO (5'-TGCAAGATTCATAAAAGTTGTCTAA-3') was purchased from Gene Tools LLC. Capped mRNAs were synthesized from $1 \mu \mathrm{g}$ of the linearized template containing WT or mutant human FOXE3 (Asp153His) by sp6 RNA polymerase using an mMESSAGE mMACHINE Kit (Ambion, Life Technologies). Synthetic mRNAs were injected into the 1-cell-stage embryos, together with the foxe $3 \mathrm{MO}$, at a dosage of $250 \mathrm{pg}$ per embryo. After injection, embryos were incubated in E3 embryo medium. Embryos were anesthetized with $0.168 \mathrm{mg} / \mathrm{ml}$ tricaine (Sigma-Aldrich), mounted in $2 \%$ methylcellulose, and photographed with a Leica fluorescence microscope equipped with a Leica DFC300FX color camera system.

Whole-mount ISH. The Foxe3-null (Foxe3--) mice were generated previously (29). Mice in this study were analyzed on a C57BL/6 mixed genetic background (Supplemental Table 2). Whole-mount ISHs were performed using standard protocols (29). Probes were labeled using digoxigenin by in vitro transcription with either T7 or T3 RNA polymerase.

Histomorphometric studies. Animals were anesthetized and perfusion fixed with $4 \%$ paraformaldehyde. The ascending and descending aortae were excised and further fixed overnight. The tissues were then embedded transversely in paraffin and cut at a thickness of $5 \mu \mathrm{m}$. Cross sections were stained with H\&E following standard protocol. The images were captured using an Olympus DP-71 camera and Olympus DP controller software (Olympus America). The thickness of the vascular walls was measured using ImageJ software (NIH). The medial area was defined as the area between the external and internal elastic lamina. The adventitia area was defined as the area between the external elastic lamina and the tunica external, the outermost layer of the vessel. The outer and inner boundaries of both vascular adventitia and media were marked with the polygon selection tool in Image J. The selections were saved in the region of interest (ROI) manager for measurement. For counting of SMC nuclei, the total number of nuclei in the medial layer from H\&E-stained slides was counted manually using the built-in cell-counter tool in Image J. Cell density was calculated as the total number of nuclei divided by the medial area. Sections were also stained with Verhoeff-van Gieson (VVG) elastin to detect the expression of elastin or Movat pentachrome to detect collagen (yellow), cells (red), elastin (black), and proteoglycans (blue). For all these area measurements, 2 sections per sample from 1 mouse were chosen at random. Data from 5 mice for each experimental group were analyzed and averaged.

TAC model. The TAC procedure was performed on 12-week-old male Foxe $3^{--}$and WT mice by staff at the Mouse Phenotype Core Laboratory at the Baylor College of Medicine using a standard surgical protocol (20). Studies were performed 2 weeks after TAC, prior to the maladaptive cardiac failure that is associated with TAC (38).

Mouse Doppler echocardiography. Successful TAC, which produces a $60 \%-80 \%$ aortic constriction, was monitored by noninvasive Doppler imaging. This procedure was performed 1 and 2 weeks after TAC. Only mice with a right carotid/left carotid flow ratio that reached a 
gradient that was more than 5- to 10 -fold higher by Doppler, indicating successful transverse constriction, were used for the studies (39). High-resolution ultrasound was performed with a Vevo 770 imaging system using a 40-MHz 704 probe (VisualSonics) as described previously (20). All scanning and analysis were performed by an experienced ultrasound technician who was blinded to the mouse genotype.

Vascular SMC isolation and culture. Mouse aortic SMCs were explanted from the ascending aortae of 4-week-old male Foxe $3^{---}$and WT mice as previously described (20). The identity of the cells as SMCs was verified by staining for anti-mouse monoclonal smooth muscle $\alpha$-actin ( $\alpha$-SMA) (Sigma-Aldrich, 95\% of cells stained positive for anti$\alpha$-SMA). SMCs were cultured in smooth muscle basal media (SmBM) containing 20\% FBS, pyruvate, HEPES, L-glutamine, antibiotic, and growth factors (SmBM Bulletkit; Lonza). Two independent cell lines were explanted from WT mice and mutant mice, using 5 mice from each group. Cells for all experiments were serum starved in SmBM containing 1\% FBS for 24 hours prior to treatment, unless otherwise noted. The results presented are representative of duplicate experiments performed on these SMCs using matched passage levels between WT and mutant SMCs, with all studies done on SMCs before passage 5 .

RNA extraction and qPCR. Total mice aortic tissues and primary vascular SMC cellular RNA were extracted with TRIzol (SigmaAldrich) according to the manufacturer's protocol. cDNA synthesis was performed using Multiscribe Reverse Transcriptase and random primers according to the manufacturer's protocol (Applied Biosystems). For qPCR analysis of mRNA expression, PCR was performed using TaqMan assays (Applied Biosystems) according to the manufacturer's instructions. TaqMan probes were purchased from Applied Biosystems and analyzed using an Applied Biosystems Prism 7900 HT Sequence Detection System according to the manufacturer's instructions. Experiments were performed in triplicate. Gapdh and Dimt1 (18S rRNA) were used as internal controls. Only qPCR data generated using the Gapdh control are shown.

IHC and immunofluorescence. Immunohistochemical staining was performed on paraffin-embedded sections with primary Abs against p-H3 (EMD Millipore; 06-570) and Mac2 (LifeSpan Biosciences; clone M3/M38), followed by a biotinylated secondary Ab according to the manufacturer's protocol. Staining was treated with a peroxidaseconjugated biotin-avidin complex using a VECTASTAIN ABC-AP Kit (Vector Laboratories) and visualized by DAB (Vector Laboratories). Slides were counterstained with hematoxylin or methyl green. For immunofluorescence staining, slides containing frozen mouse aortic cross sections (7- $\mu$ m thick) were immediately fixed with $4 \%$ paraformaldehyde for 30 minutes, blocked using $0.1 \%$ Triton $\mathrm{X}$ and $5 \%$ normal rat serum for 30 minutes at $37^{\circ} \mathrm{C}$, and then incubated overnight at $4^{\circ} \mathrm{C}$ with rat anti-macrophage (Abcam; MOMA-2; ab33451) at a concentration of 1:200. After washing, an Alexa Fluor 568-conjugated secondary Ab (Invitrogen, Life Technologies; A-21124) was added at a dilution of 1:200 for 1 hour at $37^{\circ} \mathrm{C}$. Slides were then counterstained with DAPI (Vector Laboratories).

Immunoblot analyses. Protein lysates were prepared from each mouse aortic tissue or from primary vascular SMCs following standard protocols. Briefly, aortic tissues were homogenized and lysed in ristocetin-induced platelet aggregation (RIPA) buffer (Bio-Rad) supplemented with protease inhibitor cocktail (Sigma-Aldrich). Protein $(25 \mu \mathrm{g})$ for each sample was separated on SDS-PAGE with Tris-HCl gel (Ready Gel; Bio-Rad), followed by transfer to polyvinylidene difluoride membranes (Immobilon-P; EMD Millipore). Membranes were immunoblotted with a primary $\mathrm{Ab}$ and the appropriate horseradish peroxidase-conjugated secondary Ab (Jackson ImmunoResearch Laboratories). Immunoblots were visualized by ECL (Amersham ECL Western Blotting Detection Reagents; GE Healthcare). The primary Abs used were: anti- $\alpha$-SMA (Sigma-Aldrich; A2547; 1:5,000); anti-SM myosin (Sigma-Aldrich; M7787; 1:2,000); anti-calponin (Sigma-Aldrich; C2687; 1:2,000); and anti-GAPDH (Fitzgerald Industries; 70R-49779; 1:10,000). Immunoblots were analyzed using ImageJ software.

Cell-cycle and apoptosis analyses by flow cytometry. Cell-cycle distribution and the number of apoptotic cells (detected as a sub-G1 fraction) were determined after propidium iodide (PI) staining (SigmaAldrich) and analyzed on a BD FACScan flow cytometer (39). Induction of apoptosis was quantified by measuring annexin $\mathrm{V}$-positive cells with flow cytometry using the Annexin V Apoptosis Detection Kit (BioVision). Annexin $\mathrm{V}$ binds to the cells that express phosphatidylserine on the outer layer of the membrane, and PI stains the cellular DNA in cells with a compromised, permeable membrane. This allows the discrimination between live (unstained), apoptotic (annexin V-positive), and necrotic cells (stained with both annexin V and PI).

TUNEL analysis of apoptosis in aortic tissue. Apoptosis was studied using the TUNEL assay. Apoptotic cells were detected using an in situ cell death detection kit (Roche Applied Science) as previously described (39).

p53 inhibition studies. PFT, a pharmacological p53 inhibitor, was purchased from (EMD Millipore) and diluted in PBS. An in vitro p53 inhibition study was performed by adding $10 \mu \mathrm{M}$ PFT to the cell culture media as previously described (21). Rates of programmed cell death were assessed with 3 independent in vitro assays. In each assay, $2 \times 10^{5} \mathrm{SMCs}$ in serum-free media were treated with $10 \mu \mathrm{M}$ PFT for 24 hours and then with or without $1 \mu \mathrm{mol} / \mathrm{l}$ staurosporine (Sigma-Aldrich) for another 24 hours. After 48 hours, cells were fixed and stained for cell-cycle and apoptosis analysis using the Annexin V Apoptosis Detection Kit (BioVision).

To investigate the role of p53 in Foxe3-related apoptosis, PFT was administered by i.p. injection at a dosage of $2.2 \mathrm{mg} / \mathrm{kg}$, then dissolved in PBS 1 hour before TAC and then every 48 hours. Animals were euthanized 2 weeks after the surgery, and the ascending aortic tissues were harvested for either RNA, total protein, histomorphometric analysis, or TUNEL assay, as described previously (21).

Statistics. Data represent the mean \pm SD. Statistical differences between WT and mutant mice or cells at different time points were analyzed by an unpaired, 2-tailed Student's $t$ test (for comparisons between 2 groups) or 1-way ANOVA (for comparisons between more than 2 groups, followed by the Newman-Keuls multiple comparisons test). Differences were considered statistically significant at $P$ values of 0.05 or less. Data for cell culture experiments represent 3 experiments performed in triplicate using separate cultures.

Two-point parametric linkage analysis of FOXE3 mutation and TAAD was performed using Superlink Software (40). A disease allele frequency of 0.0001 and complete penetrance in males and $70 \%$ penetrance in females with no phenocopies were used in the analysis.

Study approval. Studies involving human subjects were approved by the Committee for the Protection of Humans of the University of Texas Health Science Center at Houston and were in agreement with French bioethics laws, and approval was provided by the Comité Consultatif de Protection des Personnes dans la Recherche Biomédicale de Boulogne Billancourt, Ile de France XI, authorization no. 11008). Written informed consent was received from all participants prior 
to their inclusion in the study. All experiments with animals were approved by the IACUC of the Baylor College of Medicine and by the IACUC of the University of Texas Health Science Center at Houston.

\section{Author contributions}

SQK and OMM performed most of the experiments and contributed to experimental design, data analysis, discussion, and writing of the manuscript. DCG and ESR contributed to family recruitment and characterization and exome sequencing analysis and assisted in the preparation of the manuscript. $\mathrm{CB}$ and GJ contributed to family recruitment and DNA sequencing analysis. LG, XYD, and LYZ performed zebrafish experiments and immunoblot analyses. CLR performed mouse TAC surgeries. SKP and AMP performed and analyzed the echocardiographic data and contributed to data analysis, discussion, and writing of the manuscript. MJB, JS, and DAN provided the exome sequencing data. SQK, CSK, and XRD performed qPCR analysis. RLSC and SML contributed to statistical analysis. ECS supervised and analyzed the zebrafish experiments. MWM contributed to scientific discussion. MJ supervised the mouse studies, wrote the animal protocol, and contributed to data analysis and discussion. DMM conceptualized the project, secured funding, supervised the work, and wrote the manuscript. All authors discussed the results and commented on the manuscript.

\section{Acknowledgments}

We thank the patients and their families for participating in these studies and Lauren Mellor-Crummey for editing the manuscript. D.M. Milewicz is supported by grants from the NIH (RO1 HL109942 and P01HL110869-01); the John Ritter Foundation; the Vivian L. Smith Foundation; and the Richard T. Pisani Funds. M.W. Majesky is supported by NIH grant HL123650. Sequencing was provided by the University of Washington Center for Mendelian Genomics (UW CMG) and was funded by the National Human Genome Research Institute and NHLBI grant 1U54HG006493 (to D.A. Nickerson, J. Shendure, and M.J. Bamshad). The GenTAC Registry has been supported by US Federal Government contracts HHSN268200648199C and HHSN268201000048C from the NHLBI and the National Institute of Arthritis and Musculoskeletal and Skin Diseases. See Supplemental Acknowledgments for details on the GenTAC Investigators and the NHLBI GO ESP.

Address correspondence to: Dianna M. Milewicz, Division of Medical Genetics, Department of Internal Medicine, University of Texas Health Science Center at Houston, 6431 Fannin Street, MSB 6100, Houston, Texas 77030, USA. Phone: 713.500.6715; E-mail: Dianna.M.Milewicz@uth.tmc.edu.
1. Arribas SM, Hinek A, González MC. Elastic fibres and vascular structure in hypertension. Pharmacol Ther. 2006;111(3):771-791.

2. Stoller JZ, Epstein JA. Cardiac neural crest. Semin Cell Dev Biol. 2005;16(6):704-715.

3. Majesky MW. Developmental basis of vascular smooth muscle diversity. Arterioscler Thromb Vasc Biol. 2007;27(6):1248-1258.

4. Wasteson P, et al. Developmental origin of smooth muscle cells in the descending aorta in mice. Development. 2008;135(10):1823-1832.

5. Milewicz DM, et al. Genetic basis of thoracic aortic aneurysms and dissections: focus on smooth muscle cell contractile dysfunction. Annu Rev Genomics Hum Genet. 2008;9:283-302.

6. Humphrey JD, Milewicz DM, Tellides G, Schwartz MA. Cell biology. Dysfunctional mechanosensing in aneurysms. Science. 2014;344(6183):477-479.

7. Hiratzka LF, et al. 2010 ACCF/AHA/AATS/ ACR/ASA/SCA/SCAI/SIR/STS/SVM guidelines for the diagnosis and management of patients with Thoracic Aortic Disease: a report of the American College of Cardiology Foundation/ American Heart Association Task Force on Practice Guidelines, American Association for Thoracic Surgery, American College of Radiology, American Stroke Association, Society of Cardiovascular Anesthesiologists, Society for Cardiovascular Angiography and Interventions, Society of Interventional Radiology, Society of Thoracic Surgeons, and Society for Vascular Medicine. Circulation. 2010;121(13):e266-e369.

8. Guo DC, et al. Recurrent gain-of-function mutation in PRKG1 causes thoracic aortic aneurysms and acute aortic dissections. Am J Hum Genet. 2013;93(2):398-404.

9. Milewicz DM, Regalado ES, Shendure J, Nicker- son DA, Guo DC. Successes and challenges of using whole exome sequencing to identify novel genes underlying an inherited predisposition for thoracic aortic aneurysms and acute aortic dissections. Trends Cardiovasc Med. 2014;24(2):53-60.

10. Wada K, et al. A deletion in a cis element of Foxe3 causes cataracts and microphthalmia in ret mice. Mamm Genome. 2011;22(11-12):693-702.

11. Medina-Martinez O, Jamrich M. Foxe view of lens development and disease. Development. 2007;134(8):1455-1463.

12. Swindell EC, Zilinski CA, Hashimoto R, Shah R, Lane ME, Jamrich M. Regulation and function of foxe3 during early zebrafish development. Genesis. 2008;46(3):177-183.

13. Shi X, et al. Zebrafish foxe3: roles in ocular lens morphogenesis through interaction with pitx3. Mech Dev. 2006;123(10):761-782.

14. Jin SW, Beis D, Mitchell T, Chen JN, Stainier DY. Cellular and molecular analyses of vascular tube and lumen formation in zebrafish. Development. 2005;132(23):5199-5209.

15. Sun $\mathrm{X}$, Zhang R, Lin $\mathrm{X}, \mathrm{Xu} \mathrm{X}$. Wnt3a regulates the development of cardiac neural crest cells by modulating expression of cysteine-rich intestinal protein 2 in rhombomere 6. Circ Res. 2008;102(7):831-839.

16. Blixt A, et al. A forkhead gene, FoxE3, is essential for lens epithelial proliferation and closure of the lens vesicle. Genes Dev. 2000;14(2):245-254.

17. Conway SJ, Bundy J, Chen J, Dickman E, Rogers $\mathrm{R}$, Will BM. Decreased neural crest stem cell expansion is responsible for the conotruncal heart defects within the splotch $(\mathrm{Sp}(2 \mathrm{H})) / \operatorname{Pax} 3$ mouse mutant. Cardiovasc Res. 2000;47(2):314-328.

18. Kanzaki-Kato N, et al. Roles of forkhead transcription factor Foxc2 (MFH-1) and endothelin receptor A in cardiovascular morphogenesis. Cardiovasc Res. 2005;65(3):711-718.
19. Wei H, et al. Endothelial expression of hypoxiainducible factor 1 protects the murine heart and aorta from pressure overload by suppression of TGF- $\beta$ signaling. Proc Natl Acad Sci US A. 2012;109(19):E841-E850.

20. Kuang SQ, et al. Aortic remodeling after Transverse aortic constriction in mice is attenuated with AT1 receptor blockade. Arterioscler Thromb Vasc Biol. 2013;33(9):2172-2179.

21. Leeper NJ, et al. Loss of CDKN2B promotes p53-dependent smooth muscle cell apoptosis and aneurysm formation. Arterioscler Thromb Vasc Biol. 2013;33(1):e1-e10.

22. Zhang W, et al. The gut-enriched Kruppel-like factor (Kruppel-like factor 4) mediates the transactivating effect of $\mathrm{p} 53$ on the p21WAF1/Cip1 promoter. J Biol Chem. 2000;275(24):18391-18398.

23. Yoshida T, Kaestner KH, Owens GK. Conditional deletion of Kruppel-like factor 4 delays downregulation of smooth muscle cell differentiation markers but accelerates neointimal formation following vascular injury. Circ Res. 2008;102(12):1548-1557.

24. Reis LM, et al. FOXE3 plays a significant role in autosomal recessive microphthalmia. Am JMed Genet A. 2010;152A(3):582-590.

25. Semina EV, Brownell I, Mintz-Hittner HA, Murray JC, Jamrich M. Mutations in the human forkhead transcription factor FOXE3 associated with anterior segment ocular dysgenesis and cataracts. Hum Mol Genet. 2001;10(3):231-236.

26. Brownell I, Dirksen M, Jamrich M. Forkhead Foxe3 maps to the dysgenetic lens locus and is critical in lens development and differentiation. Genesis. 2000;27(2):81-93.

27. Saleem RA, Banerjee-Basu S, Berry FB, Baxevanis $\mathrm{AD}$, Walter MA. Analyses of the effects that disease-causing missense mutations have on the 
structure and function of the winged-helix protein FOXC1. Am J Hum Genet. 2001;68(3):627-641.

28. French CR, et al. Mutation of FOXC1 and PITX2 induces cerebral small-vessel disease. J Clin Invest. 2014;124(11):4877-4881.

29. Medina-Martinez O, Brownell I, AmayaManzanares F, Hu Q, Behringer RR, Jamrich M. Severe defects in proliferation and differentiation of lens cells in Foxe3 null mice. Mol Cell Biol. 2005;25(20):8854-8863.

30. He R, et al. Characterization of the inflammatory and apoptotic cells in the aortas of patients with ascending thoracic aortic aneurysms and dissections. J Thorac Cardiovasc Surg. 2006;131(3):671-678.

31. Clarke MC, et al. Chronic apoptosis of vascular smooth muscle cells accelerates atherosclerosis and promotes calcification and medial degeneration. Circ Res. 2008;102(12):1529-1538.
32. Habashi JP, et al. Losartan, an AT1 antagonist, prevents aortic aneurysm in a mouse model of Marfan syndrome. Science. 2006;312(5770):117-121.

33. Emrich FC, et al. Enhanced caspase activity contributes to aortic wall remodeling and early aneurysm development in a murine model of Marfan syndrome. Arterioscler Thromb Vasc Biol. 2015;35(1):146-154.

34. Komarov PG, et al. A chemical inhibitor of p53 that protects mice from the side effects of cancer therapy. Science. 1999;285(5434):1733-1737.

35. Sohn D, Graupner V, Neise D, Essmann F, Schulze-Osthoff K, Jänicke RU. Pifithrin- $\alpha$ protects against DNA damage-induced apoptosis downstream of mitochondria independent of p53. Cell Death Differ. 2009;16(6):869-878.

36. Guo DC, et al. Mutations in smooth muscle $\alpha$-actin (ACTA2) cause coronary artery disease, stroke, and moyamoya disease, along with thoracic aortic disease. Am J Hum Genet. 2009;84(5):617-627.

37. Regalado ES, et al. Exome sequencing identifies SMAD3 mutations as a cause of familial thoracic aortic aneurysm and dissection with intracranial and other arterial aneurysms. Circ Res. 2011;109(6):680-686.

38. deAlmeida AC, van Oort RJ, Wehrens XH. Transverse aortic constriction in mice.J Vis Exp. 2010;38(38):1729.

39. Kuang SQ, et al. Aberrant DNA methylation and epigenetic inactivation of Eph receptor tyrosine kinases and ephrin ligands in acute lymphoblastic leukemia. Blood. 2010;115(12):2412-2419.

40. Silberstein M, Tzemach A, Dovgolevsky N, Fishelson M, Schuster A, Geiger D. Online system for faster multipoint linkage analysis via parallel execution on thousands of personal computers. Am J Hum Genet. 2006;78(6):922-935. 